\title{
DIRAC STRUCTURES AND BOUNDARY CONTROL SYSTEMS ASSOCIATED WITH SKEW-SYMMETRIC DIFFERENTIAL OPERATORS*
}

\author{
Y. LE GORREC ${ }^{\dagger}$, H. ZWART ${ }^{\ddagger}$, AND B. MASCHKE $^{\dagger}$
}

\begin{abstract}
Associated with a skew-symmetric linear operator on the spatial domain $[a, b]$ we define a Dirac structure which includes the port variables on the boundary of this spatial domain. This Dirac structure is a subspace of a Hilbert space. Naturally, associated with this Dirac structure is an infinite-dimensional system. We parameterize the boundary port variables for which the $C_{0}$ semigroup associated with this system is contractive or unitary. Furthermore, this parameterization is used to split the boundary port variables into inputs and outputs. Similarly, we define a linear port controlled Hamiltonian system associated with the previously defined Dirac structure and a symmetric positive operator defining the energy of the system. We illustrate this theory on the example of the Timoshenko beam.
\end{abstract}

Key words. port Hamiltonian systems, strongly continuous semigroup, boundary control systems, Dirac structures

AMS subject classifications. 37K05, 35B37, 93C05, 35B30, 35G15, 47D03, 47D60

DOI. $10.1137 / 040611677$

1. Introduction. Port Hamiltonian systems have been introduced in the finitedimensional case as an analytical frame for the modeling and control of open physical systems $[12,14,18,28]$. The key concepts are the definition of pairs of power conjugated variables and the geometric structure defined on them. This geometric structure is called the Dirac structure $[2,5]$. These Dirac structures also define the internal geometric structure of the physical system as the structure of their interaction with the environment $[12,29]$. It reflects the (discrete) topology and the geometry of the physical system under consideration such as the port connection graph, constraints, or interdomain coupling $[4,15,18]$. Furthermore, it is the geometric structure which allows us to define implicit Hamiltonian systems and Hamiltonian systems with port variables [4, 27, 28]. Port Hamiltonian systems have been used for the design of stabilizing control laws; see, e.g., [13, 21, 22].

Recently, an extension of port Hamiltonian systems to infinite-dimensional systems has been proposed for distributed parameter systems with energy flow at their boundary; see $[16,30]$. The state space is a vector space of differential forms defined on the spatial domain and the port variables are defined on the boundary of the spatial domain. The port Hamiltonian system is defined with respect to a so-called StokesDirac structure, which in turn is uniquely defined by the exterior derivatives and the order of the differential forms. The Stokes-Dirac structure represents the canonical

${ }^{*}$ Received by the editors July 15, 2004; accepted for publication (in revised form) April 25, 2005; published electronically December 6, 2005.

http://www.siam.org/journals/sicon/44-5/61167.html

${ }^{\dagger}$ LAGEP, UCB Lyon 1 - CNRS UMR 5007, CPE Lyon - Bâtiment 308 G, Université Claude Bernard Lyon-1, 43, bd du 11 Novembre 1918, F-69622 Villeurbanne cedex, France (legorrec@lagep. univ-lyon1.fr, maschke@lagep.univ-lyon1.fr). The contribution of these authors has been done in the context of the European sponsored project GeoPlex with reference code IST-2001-34166. Further information is available at http://www.geoplex.cc.

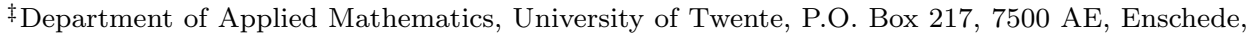
The Netherlands (h.j.zwart@math.utwente.nl). 
interdomain coupling in physical systems [19]. Finally, the Stokes-Dirac structures have been extended in order to encompass fluid dynamics and beam models [30].

Associated with linear skew-symmetric differential operators, we define Dirac structures and port Hamiltonian systems. Our definition extends the definition of Stokes-Dirac structures in which the operator needed to have differential degree one. We use an alternative definition of a Dirac structure on Hilbert spaces as proposed in [23] and [7]. In [7] Dirac structures on Hilbert spaces have also been used for the study of their composition (interconnection) and the definition of scattering representations. In this paper, we are restricting ourselves to one-dimensional spatial domains.

A major motivation of this work is to provide a theoretic formulation of open Hamiltonian systems, i.e., systems which are subject to some energy flow at their boundary. This formulation is acausal, i.e., a priori there is no distinction between inputs and outputs. The acausal formulation is obtained by first introducing boundary port variables. Second, these boundary port variables together with the (formal) skew-symmetric operator lead to the Dirac structure associated with the system.

The second motivation of this paper is to study the existence of solutions for our class of systems. This immediately implies some causality conditions among the port variables. Namely, for a (more or less) free choice of inputs there should exist a solution and the outputs should follow from it. In order to show existence of solutions, we relate our system to the class of boundary control systems. We remark that there are other general system classes which we could have chosen, e.g., system nodes [26]. We have chosen the class of boundary control systems, since this fits most naturally to our class of PDEs with their control at the boundary. As a result, we derive a parameterizing of the port variables such that the semigroup associated with the boundary control system is a contraction semigroup.

This paper is organized as follows. In section 2 we recall the definition of Dirac structures on Hilbert spaces. In section 3 we define Dirac structures associated with skew-symmetric linear differential operators and its conjugated port variables on the boundary of the spatial domain. In section 4 we associate with our Dirac structure a family of boundary control systems. The input of this boundary control system is chosen to lie in a subspace of the boundary port variables. The semigroup associated with this system is a contraction semigroup. By choosing the output to lie in the complementary of the "input subspace" we get a power balance system. The above construction gives the parameterization of all systems for which the associated semigroup is contractive and/or unitary. In section 5 we define a port Hamiltonian system associated with a skew-symmetric differential operator and with a Hamiltonian function. This Hamiltonian is a function defined by a symmetric and coercive linear operator and represents the energy in the system.

2. Dirac structures defined on Hilbert spaces. In this section, we recall the definition of Dirac structures defined on Hilbert spaces proposed by Parsian and Shafei Deh Abad in [23] and by Golo and coauthors in [7, 8]. We shall follow the definitions and notation of $[7,8]$ for the purpose of analyzing and treating the composition of Dirac structures in the frame of port-based modeling and control. This notation is borrowed partially from the bond graph language, which has been a major source of inspiration for the model definition of port Hamiltonian systems [7, 19, 18].

Let us first define the space of bond variables which is constituted of pairs of conjugated variables endowed with a pairing. For models of physical systems this corresponds to an associated instantaneous power; see [1,11]. Let the space of flow variables, denoted by $\mathcal{F}$, and the space of effort variables, denoted by $\mathcal{E}$, be real Hilbert 
spaces endowed with the inner products $\langle., .\rangle_{\mathcal{F}}$ and $\langle., .\rangle_{\mathcal{E}}$, respectively. Assume moreover that $\mathcal{F}$ and $\mathcal{E}$ are isometrically isomorphic, that is, there exists an isometry: $r_{\mathcal{F}, \mathcal{E}}: F \longrightarrow \mathcal{E}$. Denote furthermore its inverse by $r_{\mathcal{E}, \mathcal{F}}$. Define now the space of bond variables as the Hilbert space $\mathcal{B}=\mathcal{F} \times \mathcal{E}$ endowed with the natural inner product

$$
\left\langle b^{1}, b^{2}\right\rangle=\left\langle f^{1}, f^{2}\right\rangle_{\mathcal{F}}+\left\langle e^{1}, e^{2}\right\rangle_{\mathcal{E}}, \quad b^{1}=\left(f^{1}, e^{1}\right), b^{2}=\left(f^{2}, e^{2}\right) \in \mathcal{B} .
$$

In order to define a Dirac structure, let us endow the bond space $\mathcal{B}$ with a canonical symmetrical pairing, i.e., a bilinear form defined as follows:

$$
\left\langle b^{1}, b^{2}\right\rangle_{+}=\left\langle f^{1}, r_{\mathcal{E}, \mathcal{F}} e^{2}\right\rangle_{\mathcal{F}}+\left\langle e^{1}, r_{\mathcal{F}, \mathcal{E}} f^{2}\right\rangle_{\mathcal{E}}, \quad b^{1}=\left(f^{1}, e^{1}\right), b^{2}=\left(f^{2}, e^{2}\right) \in \mathcal{B} .
$$

We define a Dirac structure on the bond space $\mathcal{B}$ using this canonical pairing. Denote by $\mathcal{D}^{\perp}$ the orthogonal subspace to $\mathcal{D}$ with respect to the symmetrical pairing (2.1):

$$
\mathcal{D}^{\perp}=\left\{b \in \mathcal{B} \mid\left\langle b, b^{\prime}\right\rangle_{+}=0 \text { for all } b^{\prime} \in \mathcal{D}\right\} .
$$

Definition 2.1. A Dirac structure $\mathcal{D}$ on the bond space $\mathcal{B}=\mathcal{F} \times \mathcal{E}$ is a subspace of $\mathcal{B}$ which is maximally isotropic with respect to the canonical symmetrical pairing (2.1), i.e.,

$$
\mathcal{D}^{\perp}=\mathcal{D}
$$

One may find different examples of such Dirac structures as well as some properties concerning their representations and their composition in [7, Chapter 5]. We shall now give a canonical example of a Dirac structure in the context of the port-based modeling of physical systems. Therefore, we consider the example of a lossless vibrating string. First, we recall the port-based model structure $[19,30]$ which gives rise to the definition of a Stokes-Dirac structure on Hilbert spaces of functions with a onedimensional domain [7]. Second, we recall the formulation of the evolution equation as a port Hamiltonian system.

Example 2.2. Consider an elastic string defined on the one-dimensional spatial domain $Z=[a, b] \subset \mathbb{R}$ and subject to boundary conditions which allow some energy flow. Let us denote by $u(t, z)$ the displacement of the string at time $t$ and position $z$. Let us first recall the port Hamiltonian formulation of its dynamics. This differs from the classical formulation based on the displacement $u(t, z)$ by the choice of the state variables $[17,30]$. In this frame, the state variables are called energy variables and are chosen in such a way that the total energy of the string does not depend on their derivatives. The elastic potential energy is a function of the strain, and the energy variable is defined by

$$
\epsilon(t, z)=\frac{\partial u}{\partial z}(t, z)
$$

The associated coenergy variable is the stress given by

$$
\sigma(t, z)=T(z) \epsilon(t, z)
$$

with $T$ denoting the elasticity modulus. Hence the potential energy is the quadratic function of the strain:

$$
U(\epsilon(t, \cdot))=\frac{1}{2} \int_{a}^{b} T(z) \epsilon(t, z)^{2} d z
$$


The kinetic energy $K$ is a function of the kinetic momentum, $p(t, z)$, and it is defined by the quadratic function

$$
K(p(t, \cdot))=\frac{1}{2} \int_{a}^{b} \frac{p^{2}(t, z)}{\mu(z)} d z .
$$

The associated coenergy variable is the velocity given by

$$
v(t, z)=\frac{1}{\mu(z)} p(t, z),
$$

where $\mu$ denotes the mass density.

The dynamical model of the vibrating string is obtained by coupling the elastic energy domain and the kinetic domain through the following relations. Consider the time variation of the energy variables, called flow variables,

$$
\frac{\partial}{\partial t}\left(\begin{array}{l}
p \\
\epsilon
\end{array}\right)=\left(\begin{array}{l}
f_{K} \\
f_{U}
\end{array}\right)
$$

The canonical interdomain coupling between the elastic-potential energy and the kinetic energy relates the flow variables with the coenergy variables. This interdomain coupling is given by the differential operator [19]

$$
\left(\begin{array}{l}
f_{K} \\
f_{U}
\end{array}\right)=\left(\begin{array}{cc}
0 & \frac{\partial}{\partial z} \\
\frac{\partial}{\partial z} & 0
\end{array}\right)\left(\begin{array}{l}
v \\
\sigma
\end{array}\right)
$$

Finally, the interaction of the vibrating string through its boundary is expressed by the definition of the boundary port variables, i.e., the velocity and stress at the boundaries of the string

$$
\left(\begin{array}{l}
w_{K} \\
w_{U}
\end{array}\right)=\left(\begin{array}{cc}
I & 0 \\
0 & I
\end{array}\right)\left(\begin{array}{l}
\left.v\right|_{a, b} \\
\left.\sigma\right|_{a, b}
\end{array}\right)
$$

The canonical interdomain coupling equation (2.10) and the boundary coupling equation (2.11) actually define a Dirac structure [7, Chapter 5] called the StokesDirac structure. Let us explain this in more detail. Consider the Hilbert spaces of the flow variables $\mathcal{F}=L^{2}([a, b], \mathbb{R}) \times L^{2}([a, b], \mathbb{R}) \times \mathbb{R}^{2} \ni\left(f_{K}, f_{U}, w_{K}\right)$ and of the effort variables $\mathcal{E}=L^{2}([a, b], \mathbb{R}) \times L^{2}([a, b], \mathbb{R}) \times \mathbb{R}^{2} \ni\left(v, \sigma, w_{U}\right)$. Furthermore, endow the bond space $\mathcal{B}=\mathcal{F} \times \mathcal{E}$ with the following pairing:

$$
\begin{aligned}
& \left\langle\left(f_{K}^{1}, f_{U}^{1}, w_{K}^{1}, v^{1}, \sigma^{1}, w_{U}^{1}\right),\left(f_{K}^{2}, f_{U}^{2}, w_{K}^{2}, v^{2}, \sigma^{2}, w_{U}^{2}\right)\right\rangle_{+} \\
& =\int_{a}^{b} f_{K}^{1} v^{2} d z+\int_{a}^{b} f_{K}^{2} v^{1} d z \\
& \quad+\int_{a}^{b} f_{U}^{1} \sigma^{2} d z+\int_{a}^{b} f_{U}^{2} \sigma^{1} d z+w_{K}^{1}{ }^{T} \Lambda w_{U}^{2}+w_{K}^{2}{ }^{T} \Lambda w_{U}^{1},
\end{aligned}
$$

where $\Lambda=\left(\begin{array}{cc}I & 0 \\ 0 & -I\end{array}\right)$.

This pairing on the bond space corresponds to the general definition given in (2.1) where both the flow and the effort vector space is a product space given by $\mathcal{F}=\mathcal{F}_{(a, b)} \times \mathcal{F}_{\partial}$ and $\mathcal{E}=\mathcal{E}_{(a, b)} \times \mathcal{E}_{\partial}$, respectively. The subspace of flow variables defined on the domain $[a, b]$ is $\mathcal{F}_{(a, b)}=L^{2}([a, b], \mathbb{R}) \times L^{2}([a, b], \mathbb{R}) \ni\left(f_{K}, f_{U}\right)$ and 
the conjugated subspace of variables is $\mathcal{E}_{(a, b)}=L^{2}([a, b], \mathbb{R}) \times L^{2}([a, b], \mathbb{R}) \ni(v, \sigma)$. These Hilbert spaces are equal and hence the isometry $r_{\mathcal{F}_{(a, b)}, \mathcal{E}_{(a, b)}}$ is the identity. On the contrary, for the pairing on the boundary port variables, the matrix $\Lambda$ actually corresponds to the definition of an isometry $r_{\mathcal{F}_{\partial}, \mathcal{E}_{\partial}}$ between the boundary port spaces $\mathcal{F}_{\partial}=\mathbb{R}^{2} \ni w_{K}$ and $\mathcal{E}_{\partial}=\mathbb{R}^{2} \ni w_{U}$ endowed with the canonical Euclidean metric.

It has been shown in $[7,8]$ that $(2.10)$ and (2.11) define a Dirac structure, namely, the Stokes-Dirac structure on $\mathcal{B}$ associated with the differential operator given in (2.10). We shall denote this Dirac structure by $\mathcal{D}_{1}$.

The system of two conservation laws (2.10), with the closure equations (2.5), (2.8), and (2.9), may be rewritten as the following Hamiltonian system [20]:

$$
\frac{\partial}{\partial t}\left(\begin{array}{l}
p \\
\epsilon
\end{array}\right)=\left(\begin{array}{cc}
0 & \frac{\partial}{\partial z} \\
\frac{\partial}{\partial z} & 0
\end{array}\right)\left(\begin{array}{l}
\delta_{p} \mathcal{H} \\
\delta_{\epsilon} \mathcal{H}
\end{array}\right)
$$

where $\mathcal{H}=U+K$ denotes the Hamiltonian function corresponding to the total energy of the system and $\delta_{p} \mathcal{H}(x)=v, \delta_{\epsilon} \mathcal{H}(x)=\sigma$ denote the variational derivatives [20] of $\mathcal{H}$ with respect to the momentum $p$ and the strain $\epsilon$, respectively. This system is indeed a Hamiltonian system [20] if the differential operator in (2.12) is skew-symmetric, i.e., if the boundary variables are such that there is no energy flow at the boundary of the system:

$$
w_{K}^{1} \Lambda w_{U}^{2}+w_{K}^{2 T} \Lambda w_{U}^{1}=0 .
$$

In order to account for some energy flow at the boundary, the evolution equation (2.12) may be completed using the port boundary variables defined in (2.11), i.e., the velocity and the strain at the boundary

$$
\left(\begin{array}{l}
w_{K} \\
w_{U}
\end{array}\right)=\left(\begin{array}{cc}
I & 0 \\
0 & I
\end{array}\right)\left(\begin{array}{l}
\left.\delta_{p} \mathcal{H}\right|_{a, b} \\
\left.\delta_{\epsilon} \mathcal{H}\right|_{a, b}
\end{array}\right)
$$

The system composed of (2.12) and (2.14) defines a port Hamiltonian system with respect to the Stokes-Dirac structure. This port Hamiltonian system is generated by the Hamiltonian $\mathcal{H}[7,8,30]$ and it may be written in the following implicit way:

$$
\left(\frac{\partial p}{\partial t}, \frac{\partial \epsilon}{\partial t}, w_{K}, \delta_{p} \mathcal{H}, \delta_{\epsilon} \mathcal{H}, w_{U}\right) \in \mathcal{D}_{1}
$$

Let us briefly compare the port Hamiltonian formulation with the formulation as a PDE. The evolution equations (2.12), with the closure equations (2.9), (2.5), may also be written in the form of the wave equations (in terms of the displacement of the string):

$$
\mu \frac{\partial^{2} u}{\partial t^{2}}=\frac{\partial}{\partial z}\left(T \frac{\partial u}{\partial z}\right)
$$

The relation between the boundary conditions of this PDE and the port variables is given by

$$
\left(\begin{array}{c}
w_{K} \\
w_{U}
\end{array}\right)=\left(\begin{array}{c}
\left.v\right|_{a, b} \\
\left.\sigma\right|_{a, b}
\end{array}\right)=\left(\begin{array}{c}
\left.\frac{\partial}{\partial t} u\right|_{a, b} \\
\left.T(z) \frac{\partial u}{\partial z}\right|_{a, b}
\end{array}\right) .
$$

This shows clearly that the PDE (2.16) does not reflect the physical structure of the system in the sense that it is not written as a system of conservation laws and 
that the total energy appears clearly. Relation (2.17) shows the difference between port variables and boundary control systems in terms of physical elementary interface variables. One may not express the static equilibrium $\sigma_{\mid a, b}$ (stress) in terms of $\frac{\partial u}{\partial z}$ without knowing $T(z)$.

Finally, we compare very briefly the port Hamiltonian formulation with a classical symplectic Hamiltonian formulation (see also [17]). Using the displacement $u(z, t)$ and the velocity $v(z, t)=\frac{\partial u}{\partial t}$ as state variables, one obtains the following infinitedimensional Hamiltonian system (with energy flows being zero at the boundary):

$$
\frac{\partial x}{\partial t}=\left(\begin{array}{cc}
0 & \frac{1}{\mu} \\
-\frac{1}{\mu} & 0
\end{array}\right)\left(\begin{array}{c}
\frac{\delta H}{\delta u} \\
\frac{\delta H}{\delta v}
\end{array}\right)=\left(\begin{array}{cc}
0 & \frac{1}{\mu} \\
-\frac{1}{\mu} & 0
\end{array}\right)\left(\begin{array}{c}
-\frac{\partial}{\partial z}\left(T \frac{\partial u}{\partial z}\right) \\
\mu v
\end{array}\right),
$$

where the Hamiltonian function is

$$
H(u, v)=\int_{a}^{b}\left(\frac{1}{2} T\left(\frac{\partial u}{\partial z}\right)^{2}+\frac{1}{2} \mu v^{2}\right) d z .
$$

Contrary to the port Hamiltonian formulation, this formulation does not make the physical structure of conservation laws appear. One may furthermore note that the Hamiltonian system is defined with respect to a symplectic Poisson bracket. This bracket is not canonical (it depends on the mass distribution of the string) and cannot be extended in a canonical way to a Dirac structure including boundary variables.

This example has shown that the Stokes-Dirac structure $\mathcal{D}_{1}$, associated with the canonical interdomain coupling, is derived from a skew-symmetric differential operator of order one. In section 3, we consider a generalization of this differential operator by considering skew-symmetric operators of any order and we derive Dirac structures on Hilbert spaces from them. In Example 2.2, we have also seen how the dynamics can be defined by using the canonical Dirac structure and the Hamiltonian; namely, the dynamics lives on the Dirac structure $\mathcal{D}_{1}$ and the total energy is defined by a Hamiltonian function. In section 4, we consider energy functions which are equal to the norm of the Hilbert space. Hence there the coenergy variables and the state variables are identical. We show how to parameterize the contractive semigroups associated with the Dirac structures defined in section 3. In section 5, finally, we distinguish between the state and the coenergy variables by introducing more general Hamiltonian functions and define port Hamiltonian systems associated with skewsymmetric differential operators of any order.

3. Dirac structure associated with a skew-symmetric operator. In this section, we extend the definition of Stokes-Dirac structures to skew-symmetric differential operators of any order. Therefore, we first recall how one may extend the Stokes theorem to such operators and how the Stokes theorem induces a symmetric pairing on the boundary variables. Second, we define boundary port variables as a linear combination of the boundary variables associated with the differential operator. Using these boundary port variables, we define a bond space and a Dirac structure associated with the differential operator.

Consider the differential operator $\mathcal{J}$ of order $N$

$$
\mathcal{J} e=\sum_{i=0}^{N} P(i) \frac{d^{i} e}{d z^{i}}(z), \quad z \in[a, b]
$$


where $e \in C^{\infty}\left((a, b) ; \mathbb{R}^{n}\right)$ and $P(i), i=0, \ldots, N$, is an $n \times n$ real matrix. The formal adjoint $\mathcal{J}^{*}$ of $\mathcal{J}$ is given by

$$
\mathcal{J}^{*} e=\sum_{i=0}^{N} P(i)^{T}(-1)^{i} \frac{d^{i} e}{d z^{i}}(z), \quad z \in[a, b] .
$$

Now assume that $\mathcal{J}$ is skew-symmetric, i.e., $\mathcal{J}=-\mathcal{J}^{*}$. From the above expression of $\mathcal{J}^{*}$ we see that this is equivalent to

$$
P(i)=P(i)^{T}(-1)^{i+1} .
$$

Using this property, we show that the bilinear symmetric pairing of $e$ and $\mathcal{J} e$ depends only on the boundary values. Thus if the boundary values are zero, then $\left\langle e_{1}, \mathcal{J} e_{2}\right\rangle+\left\langle e_{2}, \mathcal{J} e_{1}\right\rangle=0$, which corresponds to the fact that $\mathcal{J}$ is (formally) skewsymmetric.

THEOREM 3.1. Let $\mathcal{J}$ be a skew-symmetric operator defined by (3.1), and let $H^{N}\left((a, b) ; \mathbb{R}^{n}\right)$ denote the Sobolev space of $N$ times differentiable functions on the interval $(a, b)$. Then for any two functions $e_{i} \in H^{N}\left((a, b) ; \mathbb{R}^{n}\right), i \in\{1,2\}$, we have that

$$
\begin{aligned}
\int_{a}^{b} e_{1}^{T}(z)\left(\mathcal{J} e_{2}\right)(z)+e_{2}^{T}(z)\left(\mathcal{J} e_{1}\right)(z) d z \\
\quad=\left[\left(e_{1}^{T}(z), \ldots, \frac{d^{N-1} e_{1}^{T}}{d z^{N-1}}(z)\right) Q\left(\begin{array}{c}
e_{2}(z) \\
\vdots \\
\frac{d^{N-1} e_{2}}{d z^{N-1}}(z)
\end{array}\right)\right]_{a}^{b}
\end{aligned}
$$

where

$$
Q=\left(Q_{i j}\right), \quad i, j=1, \ldots, N
$$

with

$$
Q_{i j}=\left\{\begin{array}{cl}
0, & i+j>N+1 \\
P(k)(-1)^{i-1}, & i+j-1=k
\end{array}\right.
$$

Furthermore, $Q$ is a symmetric matrix.

Proof. The result can easily be derived from iterative integration by parts; see [10] for details.

The above theorem shows that any skew-symmetric differential operator $\mathcal{J}$ gives rise to a symmetric bilinear product on the space of boundary conditions $e(a), \ldots$, $\frac{d^{N-1} e}{d z^{N-1}}(a), e(b), \ldots, \frac{d^{N-1} e}{d z^{N-1}}(b)$. The coefficients of this symmetric product, captured in the matrix $Q$, are uniquely defined by the coefficients of the skew-symmetric differential operator $\mathcal{J}$. In what follows, we shall define port boundary variables and a bond space in such a way that the Stokes theorem applied to the differential operator may be expressed using the canonical symmetric pairing defined in (2.1). Therefore, let us focus, in a first step, on the properties of $Q$ and define the matrix $R_{\text {ext }}$ which is used for defining the port variables. First of all, note that $Q$ has the following form:

$$
Q=\left(\begin{array}{cccccc}
P(1) & P(2) & P(3) & \ldots & P(N-1) & P(N) \\
-P(2) & -P(3) & -P(4) & \ldots & -P(N) & 0 \\
P(3) & P(4) & . \cdot & . \cdot & 0 & 0 \\
-P(4) & . \cdot & . . & . \cdot & & \vdots \\
\vdots & . \cdot & & & & \vdots \\
(-1)^{N-1} P(N) & 0 & \ldots & \ldots & \ldots & 0
\end{array}\right) .
$$


From the form of $Q$, the proof of the following lemma is immediate.

Lemma 3.2. The matrix $Q$ introduced in Theorem 3.1 is symmetric and

$$
\operatorname{ker} Q=\{0\}
$$

if and only if $\operatorname{ker} P(N)=\{0\}$.

From now on we assume that $Q$ is nonsingular.

Definition 3.3. The matrix $Q_{\text {ext }}$ in $\mathbb{R}^{2 n N \times 2 n N}$ associated with the differential operator $\mathcal{J}$ is defined by

$$
Q_{\mathrm{ext}}=\left(\begin{array}{cc}
Q & 0 \\
0 & -Q
\end{array}\right)
$$

Looking at (3.1), one can easily see that it is necessary to proceed to an appropriate change of variables to make this relation equivalent to the desired canonical symmetrical pairing defined in (2.1). This change of variables is done using the matrix $R_{\text {ext }}$ detailed in Lemma 3.4.

Lemma 3.4. The matrix $R_{\text {ext }}$ defined as

$$
R_{\mathrm{ext}}=\frac{1}{\sqrt{2}}\left(\begin{array}{cc}
Q & -Q \\
I & I
\end{array}\right)
$$

is invertible and satisfies

$$
\left(\begin{array}{cc}
Q & 0 \\
0 & -Q
\end{array}\right)=R_{\mathrm{ext}}^{T} \Sigma R_{\mathrm{ext}}
$$

where

$$
\Sigma=\left(\begin{array}{ll}
0 & I \\
I & 0
\end{array}\right)
$$

All possible matrices $R$ which satisfy (3.8) are given by the formula

$$
R=U R_{\mathrm{ext}}
$$

with $U$ satisfying $U^{T} \Sigma U=\Sigma$.

Proof. We have that

$$
\frac{1}{\sqrt{2}}\left(\begin{array}{cc}
Q & I \\
-Q & I
\end{array}\right)\left(\begin{array}{cc}
0 & I \\
I & 0
\end{array}\right)\left(\begin{array}{cc}
Q & -Q \\
I & I
\end{array}\right) \frac{1}{\sqrt{2}}=\left(\begin{array}{cc}
Q & 0 \\
0 & -Q
\end{array}\right) .
$$

Thus using the fact that $Q$ is symmetric $R_{\text {ext }}:=\frac{1}{\sqrt{2}}\left(\begin{array}{cc}Q & -Q \\ I & I\end{array}\right)$ satisfies (3.8). Since $Q$ is invertible, the invertibility of $R_{\text {ext }}$ follows from (3.8).

Let $W$ be another solution of (3.8). Hence

$$
W^{T} \Sigma W=\left(\begin{array}{cc}
Q & 0 \\
0 & -Q
\end{array}\right)=R_{\mathrm{ext}}^{T} \Sigma R_{\mathrm{ext}} .
$$

This can be written in the equivalent form

$$
R_{\text {ext }}^{-T} W^{T} \Sigma W R_{\text {ext }}^{-1}=\Sigma .
$$

Calling $W R_{\text {ext }}^{-1}=U$, we have that $U^{T} \Sigma U=\Sigma$ and $W=U R_{\text {ext }}$, which proves the assertion. 
The crucial step in defining the Dirac structure associated with the operator $\mathcal{J}$ is to define the boundary port variables. These are the following linear combinations of the boundary conditions.

DeFINITION 3.5. The boundary port variables associated with the differential operator $\mathcal{J}$ are the vectors $e_{\partial}, f_{\partial} \in \mathbb{R}^{n N}$ defined by

$$
\left(\begin{array}{c}
f_{\partial} \\
e_{\partial}
\end{array}\right)=R_{\operatorname{ext}}\left(\begin{array}{c}
e(b) \\
\vdots \\
\frac{d^{N-1} e}{d z^{N-1}}(b) \\
e(a) \\
\vdots \\
\frac{d^{N-1} e}{d z^{N-1}}(a)
\end{array}\right)
$$

where $R_{\text {ext }}$ is defined by (3.7).

Consider the effort and flow spaces $\mathcal{E}=\mathcal{F}=L^{2}\left((a, b) ; \mathbb{R}^{n}\right) \times \mathbb{R}^{n N}$ with their natural inner product. We define the bond space $\mathcal{B}$ as $\mathcal{F} \times \mathcal{E}$ with the canonical symmetrical pairing

$$
\begin{aligned}
& \left\langle\left(f^{1}, f_{\partial}^{1}, e^{1}, e_{\partial}^{1}\right),\left(f^{2}, f_{\partial}^{2}, e^{2}, e_{\partial}^{2}\right)\right\rangle_{+} \\
& \quad=\left\langle e^{1}, f^{2}\right\rangle_{L^{2}}+\left\langle e^{2}, f^{1}\right\rangle_{L^{2}}-\left\langle e_{\partial}^{1}, f_{\partial}^{2}\right\rangle-\left\langle e_{\partial}^{2}, f_{\partial}^{1}\right\rangle,
\end{aligned}
$$

where

$$
\left(f^{i}, f_{\partial}^{i}, e^{i}, e_{\partial}^{i}\right) \in \mathcal{B}, \quad i=\{1,2\} .
$$

Let us emphasize that this pairing on the bond space corresponds to the general definition given in (2.1), where the pairing on the bond space is defined modulo an isometry $r_{\mathcal{F}, \mathcal{E}}$. The space of flow variables is the product space $\mathcal{F}=L^{2}\left((a, b) ; \mathbb{R}^{n}\right) \times$ $\mathbb{R}^{N}$. Thus every flow element is a pair with the top element a function, and the bottom element is a part of the (boundary) port variable. The same description holds for the space of effort variables. The spaces $\mathcal{F}$ and $\mathcal{E}$ are equal and the natural isometry would be the identity. However, we choose

$$
r_{\mathcal{F}, \mathcal{E}}=\left(\begin{array}{cc}
I & 0 \\
0 & -I
\end{array}\right)
$$

It is easy to see that this is an isometry, which is equal to its own inverse. Furthermore, with this choice (2.1) equals (3.11).

On the bond space $\mathcal{B}$ with the symmetrical pairing (3.11) we define the Dirac structure, $\mathcal{D}_{\mathcal{J}}$, associated with the linear skew symmetric operator $\mathcal{J}$.

This Dirac structure is nothing else but the expression of the Stokes theorem (recalled in Theorem 3.1) with respect to the port variables defined in Definition 3.5.

Theorem 3.6. Let $H^{N}\left((a, b) ; \mathbb{R}^{n}\right)$ denote the Sobolev space of $N$ times differentiable functions on the interval $(a, b)$. The subspace $\mathcal{D}_{\mathcal{J}}$ of $\mathcal{B}$ defined as

$$
\mathcal{D}_{\mathcal{J}}=\left\{\left(\begin{array}{c}
f \\
f_{\partial} \\
e \\
e_{\partial}
\end{array}\right) \mid e \in H^{N}\left((a, b) ; \mathbb{R}^{n}\right), \mathcal{J} e=f,\left(\begin{array}{c}
f_{\partial} \\
e_{\partial}
\end{array}\right)=R_{\operatorname{ext}}\left(\begin{array}{c}
e(b) \\
\vdots \\
\frac{d^{N-1} e}{d z^{N-1}}(b) \\
e(a) \\
\vdots \\
\frac{d^{N-1} e}{d z^{N-1}}(a)
\end{array}\right)\right\}
$$


is a Dirac structure.

Proof. The Dirac structure is defined by the fact that $\mathcal{D}_{\mathcal{J}}=\mathcal{D}_{\mathcal{J}}^{\perp}$.

Step 1. Recall that $\mathcal{D}_{\mathcal{J}} \subset \mathcal{D}_{\mathcal{J}}^{\perp}$ is equivalent to the canonical product $\langle b, b\rangle_{+}$being zero for all $b \in \mathcal{D}_{\mathcal{J}}$. From (3.11) we have that

$$
\begin{aligned}
& \left\langle\left(f, f_{\partial}, e, e_{\partial},\right),\left(f, f_{\partial}, e, e_{\partial}\right)\right\rangle_{+} \\
& \quad=\langle e, \mathcal{J} e\rangle_{L^{2}}+\langle e, \mathcal{J} e\rangle_{L^{2}}-e_{\partial}^{T} f_{\partial}-e_{\partial}^{T} f_{\partial} \\
& \quad=\left[\left(e^{T}(z), \ldots, \frac{d^{N-1} e^{T}}{d z^{N-1}}(z)\right) Q\left(\begin{array}{c}
e(z) \\
\vdots \\
\frac{d^{N-1} e}{d z^{N-1}}(z)
\end{array}\right)\right]_{a}^{b}-2 e_{\partial}^{T} f_{\partial} \\
& =\left(e^{T}(b), \ldots, \frac{d^{N-1} e^{T}}{d z^{N-1}}(a)\right)\left(\begin{array}{cc}
Q & 0 \\
0 & -Q
\end{array}\right)\left(\begin{array}{c}
e(b) \\
\vdots \\
\frac{d^{N-1}}{d z^{N-1}}(a)
\end{array}\right)-2 e_{\partial}^{T} f_{\partial} \\
& =\left(f_{\partial}^{T}, e_{\partial}^{T}\right) \Sigma\left(\begin{array}{c}
f_{\partial} \\
e_{\partial}
\end{array}\right)-2 e_{\partial}^{T} f_{\partial}=0,
\end{aligned}
$$

where we have used Theorem 3.1 and (3.8).

Step 2. Let $\left(\phi, \phi_{\partial}, \varepsilon, \varepsilon_{\partial}\right) \in \mathcal{D} \frac{\perp}{\mathcal{J}}$. Choose $e \in H^{N}\left((a, b) ; \mathbb{R}^{n}\right)$ with compact support strictly included in $(a, b)$. Thus $\frac{d^{k} e}{d z^{k}}, k \in\{0, \ldots, N-1\}$, are zero in $a$ and $b$. Then it is easy to see that $(\mathcal{J} e, 0, e, 0) \in \mathcal{D}_{\mathcal{J}}$. Using (3.11) we have

$$
0=\langle e, \phi\rangle+\langle\varepsilon, f\rangle=\langle e, \phi\rangle+\langle\varepsilon, \mathcal{J} e\rangle
$$

for all such $e$. This implies that $\varepsilon \in H^{N}\left((a, b) ; \mathbb{R}^{n}\right)$ and $\mathcal{J} \varepsilon=\phi$.

Step 3. Let $\left(\phi, \phi_{\partial}, \varepsilon, \varepsilon_{\partial}\right) \in \mathcal{D}_{\mathcal{J}}^{\perp}$ and let $\left(f, f_{\partial}, e, e_{\partial}\right) \in \mathcal{D}_{\mathcal{J}}$. From Step 2 and (3.11) we obtain

$$
\begin{aligned}
0 & =\langle e, \mathcal{J} \varepsilon\rangle+\langle\varepsilon, \mathcal{J} e\rangle-e_{\partial}^{T} \phi_{\partial}-\varepsilon_{\partial}^{T} f_{\partial} \\
& =\left[\left(e^{T}(z), \ldots, \frac{d^{N-1} e^{T}(z)}{d z^{N-1}}(z)\right) Q\left(\begin{array}{c}
\varepsilon(z) \\
\vdots \\
\frac{d^{N-1} \varepsilon(z)}{d z^{N-1}}(z)
\end{array}\right)\right]_{a}^{b}-e_{\partial}^{T} \phi_{\partial}-\varepsilon_{\partial}^{T} f_{\partial} \\
& =\left(f_{\partial}^{T}, e_{\partial}^{T}\right) \Sigma R_{\operatorname{ext}}\left(\begin{array}{c}
\varepsilon(b) \\
\vdots \\
\frac{d^{N-1} \varepsilon}{d z^{N-1}}(a)
\end{array}\right)-e_{\partial}^{T} \phi_{\partial}-\varepsilon_{\partial}^{T} f_{\partial} \\
& =\left(e_{\partial}^{T}, f_{\partial}^{T}\right)\left[R_{\operatorname{ext}}\left(\begin{array}{c}
\varepsilon(b) \\
\vdots \\
\frac{d^{N-1} \varepsilon}{d z^{N-1}}(a)
\end{array}\right)-\left(\begin{array}{c}
\phi_{\partial} \\
\varepsilon_{\partial}
\end{array}\right)\right] .
\end{aligned}
$$

By a proper choice of $e$, we can let the vectors $e_{\partial}$ and $f_{\partial}$ have arbitrary values. Thus the above equality has to hold for all $e_{\partial} \in \mathbb{R}^{n N}$ and $f_{\partial} \in \mathbb{R}^{n N}$. Consequently, we have that

$$
R_{\mathrm{ext}}\left(\begin{array}{c}
\varepsilon(b) \\
\vdots \\
\frac{d^{N-1} \varepsilon}{d z^{N-1}}(a)
\end{array}\right)=\left(\begin{array}{c}
\phi_{\partial} \\
\varepsilon_{\partial}
\end{array}\right) .
$$

In conclusion, we have that $\mathcal{D}_{\mathcal{J}}=\mathcal{D}_{\mathcal{J}}^{\perp}$, and so $\mathcal{D}_{\mathcal{J}}$ is a Dirac structure. 
4. Contraction semigroups, boundary control systems, and their parameterization. In the previous section we have associated with the skew-symmetric operator $\mathcal{J}$ a Dirac structure $\mathcal{D}_{\mathcal{J}}$. In this section, we shall define dynamic systems with inputs, states, and outputs with respect to this Dirac structure. These systems will be boundary control systems in the sense of the semigroup theory [3], which implies that the controls and observations act on the boundary of the spatial domain. With respect to the Dirac structure $\mathcal{D}_{\mathcal{J}}$ it is possible to define many systems. However, we consider only those systems for which the energy does not grow when the input is zero. This implies that the associated semigroup is contractive. We parameterize all these systems by $n N$-dimensional linear subspaces of the port variables. As a consequence of this parameterization, we identify those systems for which the associated semigroup is unitary.

We begin by showing that $\mathcal{J}$ is the infinitesimal generator of a contraction semigroup for appropriate choices of the boundary conditions.

4.1. Contraction semigroups associated with $\mathcal{D}_{\mathcal{J}}$. We begin by studying the differential operator $\mathcal{J}$ for different boundary conditions. As stated above, we want to characterize those boundary conditions for which the associated differential operator is the infinitesimal generator of a strongly continuous semigroup. Furthermore, this semigroup must be contractive, i.e., $\|T(t) e\| \leq\|e\|$ for all $t \geq 0$ and $e \in L^{2}\left((a, b) ; \mathbb{R}^{n}\right)$. We obtain this characterization of the boundary conditions by using Theorem 3.1.6 of [9]. For the history of this result, we refer to the bibliographical comments at the end of [9].

Before stating this result, we recall the following parameterization. Let $\Sigma=\left(\begin{array}{ll}0 & I \\ I & 0\end{array}\right)$. A full rank matrix $W$ of size $n N \times 2 n N$ satisfies

$$
W \Sigma W^{T} \geq 0
$$

if and only if

$$
W=S(I+V \quad I-V)
$$

with $S$ an invertible matrix, and $V$ satisfying $V V^{T} \leq I$; see the appendix for a proof.

In the following theorem it is shown that if the port variables are restricted to the kernel of $W$, then this defines the domain of a contraction semigroup associated with the operator $\mathcal{J}$.

Theorem 4.1. Let $W$ be a full rank matrix of size $k \times 2 n N$. Define the operator $J_{W}$ and its domain, $D\left(J_{W}\right)$, as

$$
J_{W} e=\mathcal{J} e
$$

and

$$
\begin{aligned}
D\left(J_{W}\right)=\left\{e \in L^{2}\left((a, b), \mathbb{R}^{n}\right) \mid \text { the port variable associated with } e,\right. \\
\\
\left(\begin{array}{c}
f_{\partial} \\
e_{\partial}
\end{array}\right), \text { is in } \text { ker } W \text { and there exists } \\
\text { an } \left.f \in L^{2}\left((a, b) ; \mathbb{R}^{n}\right) \text { such that }\left(f, f_{\partial}, e, e_{\partial}\right) \in \mathcal{D}_{\mathcal{J}}\right\} .
\end{aligned}
$$

Then $J_{W}$ generates a contraction semigroup $(T(t))_{t \geq 0}$ on $L^{2}\left((a, b) ; \mathbb{R}^{n}\right)$ if and only if $k=n N$ and (4.1) holds. 
Furthermore, $J_{W}$ is the infinitesimal generator of a unitary semigroup on $L^{2}\left((a, b) ; \mathbb{R}^{n}\right)$ if and only if $k=n N$ and $W \Sigma W^{T}=0$.

Proof. It is well known that operator $A$ is the infinitesimal generator of a contraction semigroup if and only if it is maximally dissipative, i.e., it is dissipative:

$$
\operatorname{Re}\langle A z, z\rangle \leq 0
$$

for all $z \in D(A)$, and it is not a proper restriction of any other dissipative operator $[24]$.

In [9] a characterization of maximal dissipative differential operators in terms of their boundary conditions is given. However, their formulation is not precisely the one we are using. Hence in the next step we relate their notation with ours. Once that is done, the proof of the theorem is straightforward.

Step 1. Define on $D(\mathcal{J})=H^{N}\left((a, b), \mathbb{C}^{n}\right)$ the operator

$$
\mathcal{A}_{0}^{*}=i \mathcal{J} \text {. }
$$

Then it is easy to see that $\mathcal{A}_{0}=i \mathcal{J}$ with $D\left(\mathcal{A}_{0}\right)=\left\{e \in H^{N}\left((a, b), \mathbb{C}^{n}\right) \mid \frac{d^{N-1} e}{d z^{N-1}}(b)\right.$ $=\cdots e(b)=0$ and $\left.\frac{d^{N-1} e}{d z^{N-1}}(a)=\cdots e(a)=0\right\}$.

Using Theorem 3.1 and Definition 3.6 we have for all $e_{1}, e_{2} \in D(\mathcal{J})$

$$
\begin{aligned}
\left\langle\mathcal{A}_{0}^{*} e_{1}, e_{2}\right\rangle-\left\langle e_{1}, \mathcal{A}_{0}^{*} e_{2}\right\rangle & =i\left\langle\mathcal{J} e_{1}, e_{2}\right\rangle+i\left\langle e_{1}, \mathcal{J} e_{2}\right\rangle \\
& =i\left\langle\left(\begin{array}{c}
e_{1}(b) \\
\vdots \\
\frac{d^{N-1} e_{1}}{d z^{N-1}}(a)
\end{array}\right), Q_{\mathrm{ext}}\left(\begin{array}{c}
e_{2}(b) \\
\vdots \\
\frac{d^{N-1} e_{2}}{d z^{N-1}}(a)
\end{array}\right)\right\rangle_{\mathbb{C}^{2 n N}} \\
& =i\left\langle\left(\begin{array}{c}
f_{1, \partial} \\
e_{1, \partial}
\end{array}\right), \Sigma\left(\begin{array}{c}
f_{2, \partial} \\
e_{2, \partial}
\end{array}\right)\right\rangle_{\mathbb{C}^{2 n N}} \\
& =i\left[\left\langle f_{1, \partial}, e_{2, \partial}\right\rangle_{\mathbb{C}^{n N}}+\left\langle e_{1, \partial}, f_{2, \partial}\right\rangle_{\mathbb{C}^{n N}}\right]
\end{aligned}
$$

where we have used (3.10). Define operators $\Gamma_{1}$ and $\Gamma_{2}$ from $H^{N}\left((a, b), \mathbb{C}^{n}\right)$ to $\mathbb{C}^{n N}$ as

$$
\Gamma_{1} e=i f_{\partial}, \quad \Gamma_{2} e=e_{\partial} .
$$

It is clear that these mappings are onto. Furthermore, we have that

$$
\left\langle\mathcal{A}_{0}^{*} e_{1}, e_{2}\right\rangle-\left\langle e_{1}, \mathcal{A}_{0}^{*} e_{2}\right\rangle=\left\langle\Gamma_{1} e_{1}, \Gamma_{2} e_{2}\right\rangle_{\mathbb{C}^{n N}}-\left\langle\Gamma_{2} e_{1}, \Gamma_{1} e_{2}\right\rangle_{\mathbb{C}^{n N}} .
$$

Step 2. Using (4.6), Theorem 3.1.6 of [9] characterizes all maximally accumulative extensions of $\mathcal{A}_{0}$. An operator $A$ is defined to be accumulative if $\operatorname{Im}\langle A z, z\rangle \leq 0$ for all $z \in D(A)$. It is maximally accumulative, when it has no nontrivial accumulative extension. It is easy to see that $A$ is a maximal accumulative extension of $\mathcal{A}_{0}$ if and only if $-i A$ is a maximal dissipative extension of $\mathcal{J}$ with the domain $D\left(\mathcal{A}_{0}\right)$.

Step 3. Theorem 3.1.6 of [9] states that any maximally accumulative extension of $\mathcal{A}_{0}$ is given by

$$
A_{K}=\mathcal{A}_{0}^{*}
$$

with

$$
D\left(A_{K}\right)=\left\{e \in D\left(\mathcal{A}_{0}^{*}\right) \mid(K-I) \Gamma_{1} e-i(K+I) \Gamma_{2} e=0\right\},
$$


where $K: \mathbb{C}^{n N} \mapsto \mathbb{C}^{n N}$ with $\|K\| \leq 1$. Using the result obtained in the previous step and (4.5), we see that any maximal dissipative extension of $\mathcal{J}, D\left(\mathcal{A}_{0}\right)$, is given by

$$
-i A_{K}=-i \mathcal{A}_{0}^{*}=\mathcal{J}
$$

with the domain

$$
\begin{aligned}
D\left(A_{K}\right) & =\left\{e \in D\left(\mathcal{A}_{0}^{*}\right) \mid(K-I) \Gamma_{1} e-i(K+I) \Gamma_{2} e=0\right\} \\
& =\left\{e \in H^{N}\left((a, b), \mathbb{C}^{n}\right) \mid(K-I) f_{\partial}-(K+I) e_{\partial}=0\right\},
\end{aligned}
$$

where $K: \mathbb{C}^{n N} \mapsto \mathbb{C}^{n N}$ with $\|K\| \leq 1$. Using Lemma A.1 we see that $-i A_{K}=J_{W}$ with $W=S(I-K, I+K)$, where $S$ is an arbitrary, invertible matrix. Since we are interested only in real conditions, we have to take $K$ real valued. Hence we have obtained a complete characterization of all boundary conditions for which the differential operator is the infinitesimal generator of a contraction semigroup.

Step 4 . The proof of the unitary case is done very similarly.

One may wonder why we have parameterized the boundary port variables using the $W$ instead of the $V$, since if two $W$ 's have the same $V$, then the associated semigroups are the same. In the following subsection, the boundary variables are decomposed into inputs and outputs. For this splitting the $W$ is important. More specifically, different $W$ 's lead to different systems, although the semigroup may be the same.

4.2. Boundary control system and port conjugated output. In the previous subsection we have derived the family of contraction semigroups from the Dirac structure $\mathcal{D}_{\mathcal{J}}$ associated with a skew-symmetric differential operator $\mathcal{J}$. More precisely, we have parameterized these semigroups by a family of subspaces of the port boundary variables defined as the kernel of a class of matrices $W$ (matrices of size $n N \times 2 n N$ satisfying (4.1)). In the following theorem, we use this $W$ to define boundary inputs/controls. Since the rank of $W$ is $n N$ and since we have $2 n N$ boundary variables, we see that we use half of the set of boundary variables to define inputs. We show that the other half may be regarded as outputs. Note that the terms input and output are used here to make the relation with infinite-dimensional systems theory. It does not necessarily mean that the input is completely free, i.e., it can be chosen arbitrarily in $L_{\text {loc }}^{2}\left((0, \infty) ; \mathbb{R}^{n N}\right)$, nor does it imply that for every initial condition in $L^{2}\left((a, b) ; \mathbb{R}^{n}\right)$ the output is well defined. The system class which is considered is the class of boundary control systems. For more information on this class, we refer the reader to section 3.3 of [3].

Using the splitting of the boundary ports into inputs and outputs, we consider in Theorems 4.4 and 4.6 some special choices which lead to classical power balance equations in the so-called impedance and scattering variables form.

TheOREM 4.2. For the differential operator $\mathcal{J}$ and the associated Dirac structure $\mathcal{D}_{\mathcal{J}}$ (see Theorem 3.6), we consider the dynamical system

$$
\left(\dot{x}(t), f_{\partial}(t), x(t), e_{\partial}(t)\right) \in \mathcal{D}_{\mathcal{J}}, \quad t \geq 0
$$

where $\left(f_{\partial}(t), e_{\partial}(t)\right)$ are the boundary port variables associated with $x(t)$; see Definition 3.5 .

Let $W$ be a full rank matrix of size $n N \times 2 n N$ satisfying (4.1), and define $\mathcal{B}$ : $H^{N}\left((a, b), \mathbb{R}^{n}\right) \rightarrow \mathbb{R}^{n N}$ as

$$
\mathcal{B} x(t):=W\left(\begin{array}{l}
f_{\partial}(t) \\
e_{\partial}(t)
\end{array}\right) .
$$


Then system (4.7) with the input defined as

$$
u(t)=\mathcal{B} x(t)
$$

is a boundary control system.

Furthermore, let $\tilde{W}$ be a full rank matrix of size $n N \times 2 n N$ with $(\underset{\tilde{W}}{W})$ invertible. If we define the linear mapping $\mathcal{C}: H^{N}\left((a, b), \mathbb{R}^{n}\right) \rightarrow \mathbb{R}^{n N}$ as

$$
\mathcal{C} x(t):=\tilde{W}\left(\begin{array}{l}
f_{\partial}(t) \\
e_{\partial}(t)
\end{array}\right)
$$

and the output as

$$
y(t)=\mathcal{C} x(t),
$$

then for $u \in C^{2}\left((0, \infty) ; \mathbb{R}^{n N}\right), x(0) \in H^{N}\left((a, b), \mathbb{R}^{n}\right)$, and $\mathcal{B} x(0)=u(0)$, the following balance equation is satisfied:

$$
\frac{1}{2} \frac{d}{d t}\|x(t)\|^{2}=\frac{1}{2}\left(u^{T}(t) y^{T}(t)\right) P_{W, \tilde{W}}\left(\begin{array}{l}
u(t) \\
y(t)
\end{array}\right),
$$

where

$$
P_{W, \tilde{W}}^{-1}=\left(\begin{array}{ll}
W \Sigma W^{T} & W \Sigma \tilde{W}^{T} \\
\tilde{W} \Sigma W^{T} & \tilde{W} \Sigma \tilde{W}^{T}
\end{array}\right)
$$

Furthermore, we have that the matrix $\left(\begin{array}{cc}W \Sigma W^{T} & W \Sigma \tilde{W}^{T} \\ \tilde{W} \Sigma W^{T} & \tilde{W} \Sigma \tilde{W}^{T}\end{array}\right)$ is invertible if and only if $\left(\begin{array}{c}W \\ \tilde{W}\end{array}\right)$ is invertible.

REMARK 4.3. The system defined by (4.7)-(4.9) may be equivalently written in the more usual form of a boundary control system:

$$
\begin{aligned}
& \dot{x}(t)=\mathcal{J} x(t), \\
& \mathcal{B} x(t)=u(t) .
\end{aligned}
$$

Proof of Theorem 4.2. In Steps 1 and 2 we show that we have a boundary control system. In Steps 3 and 4, we prove (4.12) and (4.13), respectively. For a boundary control system we have to show that for zero inputs, the system is a $C_{0}$-semigroup, and furthermore that there exists a bounded operator $B$ mapping onto the domain of $\mathcal{B}$ and such that $\mathcal{B B} u=u$ for all $u \in \mathbb{R}^{n N}$.

Step 1 . As mentioned above, we have to show that $J_{W}$ defined as

$$
J_{W} x=\mathcal{J} x
$$

on

$$
D\left(J_{W}\right)=D(\mathcal{J}) \cap \operatorname{ker} \mathcal{B}
$$

is an infinitesimal generator. This follows directly from Theorem 4.1.

Step 2. We have to find a bounded linear operator $B$ such that $B u \in D(\mathcal{B})=$ $H^{N}\left((a, b) ; \mathbb{R}^{n N}\right)$ and $\mathcal{B} B u=u$ for all $u \in \mathbb{R}^{n N}$.

Let $\left\{u^{1}, \ldots, u^{n N}\right\}$ be the standard basis of the input space $\mathbb{R}^{n N}$, i.e., $u^{i}=$ $\left(\delta_{i j}\right)_{j=1, \ldots, n N}^{T}$. Since $R_{\text {ext }}$ is invertible, and since $W$ has rank $n N$ there exists for every $u^{i}$ a $v^{i} \in \mathbb{R}^{2 n N}$ such that

$$
W R_{\mathrm{ext}} v^{i}=u^{i} .
$$


Let $v_{k}^{i}$ denote the $k$ th block of $v^{i}, k=1, \ldots, 2 N$. Using functions $f_{r, j}$ and $f_{l, j}$ introduced in Lemma A.3, we define the $i$ th column of $B$ as

$$
B_{i}=\sum_{k=1}^{N} v_{k}^{i} f_{r, k-1}(z)+\sum_{k=1}^{N} v_{k+N}^{i} f_{l, k-1}(z) .
$$

It is straightforward that $B$ is a bounded operator mapping onto the domain of $\mathcal{J}$. Furthermore, by Definition 3.5 we have that

$$
\mathcal{B} B_{i}=W R_{\operatorname{ext}}\left(\begin{array}{c}
B_{i}(b) \\
\vdots \\
\frac{d^{N-1} B_{i}(b)}{d z^{N-1}} \\
B_{i}(a) \\
\vdots \\
\frac{d^{N-1} B_{i}(a)}{d z^{N-1}}
\end{array}\right) .
$$

Now by definition

$$
\frac{d^{p} B_{i}}{d z^{p}}(z)=\sum_{k=1}^{N} v_{k}^{i} f_{r, k-1}^{(p)}(z)+\sum_{k=1}^{N} v_{k+N}^{i} f_{l, k-1}^{(p)}(z) .
$$

From (A.3) and (A.4) of Lemma A.3, we have that

$$
\frac{d^{p} B_{i}}{d z^{p}}(b)=v_{p+1}^{i} \quad \text { and } \quad \frac{d^{p} B_{i}}{d z^{p}}(a)=v_{p+N+1}^{i},
$$

and so $B$ satisfies

$$
\mathcal{B} B u=W R_{\text {ext }}\left(\begin{array}{c}
v_{1}^{i} \\
\vdots \\
v_{2 N}^{i}
\end{array}\right)=u^{i}
$$

Step 3. By the definition of $B$ and $D\left(J_{W}\right)$, we see that the conditions stated in the theorem are the same as $x(0)-B u(0) \in D\left(J_{W}\right)$. Hence by Theorem 3.3.3 of [3] we have that there exists a classical solution of (4.7)-(4.9). Hence, in particular, $x(t) \in H^{N}\left((a, b), \mathbb{R}^{n}\right)$ holds pointwise in $t, x(t)$ is differentiable as a function of $t$, and $\dot{x}(t)=\mathcal{J} x(t)$. Using this, we obtain

$$
\begin{aligned}
& \frac{d}{d t}\|x(t)\|^{2}=\frac{d}{d t}\langle x(t), x(t)\rangle \\
& =\langle\dot{x}(t), x(t)\rangle+\langle x(t), \dot{x}(t)\rangle \\
& =\langle\mathcal{J} x(t), x(t)\rangle+\langle x(t), \mathcal{J} x(t)\rangle \\
& =\left(\begin{array}{ll}
f_{\partial}^{T}(t) & e_{\partial}^{T}(t)
\end{array}\right) \Sigma\left(\begin{array}{l}
f_{\partial}(t) \\
e_{\partial}(t)
\end{array}\right) .
\end{aligned}
$$

On the other hand, we have that

$$
\left(\begin{array}{l}
u \\
y
\end{array}\right)=\left(\begin{array}{l}
W \\
\tilde{W}
\end{array}\right)\left(\begin{array}{l}
f_{\partial} \\
e_{\partial}
\end{array}\right)
$$


Combining this with (4.16) gives that

$$
\begin{aligned}
& \frac{d}{d t}\|x(t)\|^{2}=\left(\begin{array}{ll}
u^{T}(t) & y^{T}(t)
\end{array}\right)\left(\begin{array}{c}
W \\
\tilde{W}
\end{array}\right)^{-T} \Sigma\left(\begin{array}{c}
W \\
\tilde{W}
\end{array}\right)^{-1}\left(\begin{array}{c}
u(t) \\
y(t)
\end{array}\right) \\
& =\left(\begin{array}{ll}
u^{T}(t) & y^{T}(t)
\end{array}\right) P_{W, \tilde{W}}\left(\begin{array}{l}
u(t) \\
y(t)
\end{array}\right) .
\end{aligned}
$$

Hence we have proved (4.12).

Step 4 . By the definition of $P_{W, \tilde{W}}$, we see that

$$
P_{W, \tilde{W}}^{-1}=\left(\begin{array}{c}
W \\
\tilde{W}
\end{array}\right) \Sigma\left(\begin{array}{c}
W \\
\tilde{W}
\end{array}\right)^{T}=\left(\begin{array}{ll}
W \Sigma W^{T} & W \Sigma \tilde{W}^{T} \\
\tilde{W} \Sigma W^{T} & \tilde{W} \Sigma \tilde{W}^{T}
\end{array}\right)
$$

which shows (4.13). From this equality the last assertion of the theorem follows directly.

Now we consider two particular cases which are canonical. For the first choice of inputs and outputs, the system becomes a lossless system. For the second choice of inputs and outputs, the balance (4.12) becomes canonical for scattering variables. We begin by characterizing the case when the boundary control system becomes a lossless system.

THEOREM 4.4. Let $W$ and $\tilde{W}$ be $n N \times 2 n N$ matrices with $W$ having full rank and satisfying (4.1). Associate with these matrices the following system:

$$
\begin{aligned}
\dot{x}(t) & =\mathcal{J} x(t), \\
u(t) & =W\left(\begin{array}{l}
f_{\partial}(t) \\
e_{\partial}(t)
\end{array}\right), \\
y(t) & =\tilde{W}\left(\begin{array}{l}
f_{\partial}(t) \\
e_{\partial}(t)
\end{array}\right),
\end{aligned}
$$

where $\left(f_{\partial}(t), e_{\partial}(t)\right)$ are the boundary port variables associated with $x(t)$; see Definition 3.5.

The above system is a boundary control system. Furthermore, it satisfies for all $u \in C^{2}\left((0, \infty) ; \mathbb{R}^{n N}\right), x(0) \in H^{N}\left((a, b) ; \mathbb{R}^{n}\right)$ with $u(0)=W\left(\begin{array}{c}f_{\partial}(0) \\ e_{\partial}(0)\end{array}\right)$ the balance equation

$$
\frac{1}{2} \frac{d}{d t}\|x(t)\|^{2}=u(t)^{T} y(t)
$$

if and only if the following conditions are satisfied:

$$
\begin{gathered}
W=S(I+V \quad I-V) \quad \text { with } S \text { invertible and } V \text { unitary } \\
\tilde{W}=\tilde{S}(I+\tilde{V} \quad I-\tilde{V}) \quad \text { with } \tilde{S} \text { invertible and } \tilde{V} \text { unitary } \\
I=2 \tilde{S}\left(I-\tilde{V} V^{T}\right) S^{T}
\end{gathered}
$$

Furthermore, under condition (4.23) the associated semigroup is unitary.

Proof. Looking at Theorem 4.2 we see that we only have to check that $P_{W, \tilde{W}}$ equals $\left(\begin{array}{ll}0 & I \\ I & 0\end{array}\right)$. By (4.13) this is equivalent to $W \Sigma W^{T}=\tilde{W} \Sigma \tilde{W}^{T}=0$ and $\tilde{W} \Sigma W^{T}=I$. By Lemma A.1 we see that the first conditions are equivalent to (4.23) and (4.24), respectively. Direct calculation gives that $\tilde{W} \Sigma W^{T}=I$ is the same as (4.25). 
Taking in the above theorem, $V=I, \tilde{V}=-I$, and $S=\tilde{S}=\frac{1}{2} I$, we obtain the following special case.

COROLlaRY 4.5. Under the general conditions as stated in Theorem 4.4 consider the system defined by

$$
\begin{aligned}
\dot{x}(t) & =\mathcal{J} x(t), \\
u(t) & =f_{\partial}(t), \\
y(t) & =-e_{\partial}(t),
\end{aligned}
$$

where $\left(f_{\partial}(t), e_{\partial}(t)\right)$ are the boundary port variables associated with $x(t)$; see Definition 3.5 .

The above system is a boundary control system with the associated semigroup unitary. Furthermore, it satisfies for all $u \in C^{2}\left((0, \infty) ; \mathbb{R}^{n N}\right), x(0) \in H^{N}\left((a, b) ; \mathbb{R}^{n}\right)$, and $u(0)=f_{\partial}(0)$ the following balance equation:

$$
\frac{1}{2} \frac{d}{d t}\|x(t)\|^{2}=u(t)^{T} y(t)
$$

In the following theorem we characterize the scattering case.

TheOREM 4.6. Let $W$ and $\tilde{W}$ be $n N \times 2 n N$ matrices with $W$ having full rank and satisfying (4.1). Associate with these matrices the following system:

$$
\begin{aligned}
\dot{x}(t) & =\mathcal{J} x(t), \\
u(t) & =W\left(\begin{array}{l}
f_{\partial}(t) \\
e_{\partial}(t)
\end{array}\right), \\
y(t) & =\tilde{W}\left(\begin{array}{l}
f_{\partial}(t) \\
e_{\partial}(t)
\end{array}\right),
\end{aligned}
$$

where $\left(f_{\partial}(t), e_{\partial}(t)\right)$ are the boundary port variable associated with $x(t)$; see Definition 3.5 .

The above system is a boundary control system. Furthermore, it satisfies for all $u \in C^{2}\left((0, \infty) ; \mathbb{R}^{n N}\right), x(0) \in H^{N}\left((a, b) ; \mathbb{R}^{n}\right)$ with $u(0)=W\left(\begin{array}{c}f_{\partial}(0) \\ e_{\partial}(0)\end{array}\right)$ the balance equation

$$
\frac{1}{2} \frac{d}{d t}\|x(t)\|^{2}=\|u(t)\|^{2}-\|y(t)\|^{2}
$$

if and only if the following conditions are satisfied:

$$
\begin{gathered}
W=S(I+V \quad I-V) \quad \text { with } 4 S\left(I+V V^{T}\right) S^{T}=I, \\
\tilde{W}=\tilde{S}\left(-I-V^{T} \quad I-V^{T}\right) \quad \text { with } 4 \tilde{S}\left(I-V^{T} V\right) \tilde{S}^{T}=I .
\end{gathered}
$$

Proof. Looking at Theorem 4.2 we see that we only have to check that $P_{W, \tilde{W}}$ equals $\left(\begin{array}{cc}2 I & 0 \\ 0 & -2 I\end{array}\right)$. By (4.13) this is equivalent to $W \Sigma W^{T}=\frac{1}{2} I, \tilde{W} \Sigma \tilde{W}^{T}=-\frac{1}{2} I$, and $W \Sigma \tilde{W}^{T}=0$.

It is easy to show that if both (4.34) and (4.35) hold, then (4.33) holds. So it remains to show the converse. Using the standard representation of $W$ (see (4.2)), we get that $W \Sigma W^{T}=\frac{1}{2} I$ is equivalently written as (4.34).

Since $\tilde{W} \Sigma \tilde{W}^{T}=-\frac{1}{2} I$, we see that $\tilde{W}$ is of full rank. Furthermore, from the relation $W \Sigma \tilde{W}^{T}=0$, we obtain that the range of $\Sigma \tilde{W}^{T}$ is contained in the kernel of 
$W$. By Lemma A.2 we have that the kernel of $W$ equals the range of $\left(\begin{array}{c}I-V \\ -I-V\end{array}\right)$. Since both the range of this matrix and that of $\Sigma \tilde{W}^{T}$ is of dimension $n N$, we find that

$$
\Sigma \tilde{W}^{T}=\left(\begin{array}{c}
I-V \\
-I-V
\end{array}\right) \tilde{S}^{T}
$$

for some invertible $\tilde{S}$. Hence we have shown that the representation of (4.35) holds. The last part of this equation follows directly from the fact that $\tilde{W} \Sigma \tilde{W}^{T}=$ $-\frac{1}{2} I$.

Choosing in the above theorem $V=0$ and $S=\tilde{S}=\frac{1}{2} I$ gives the following corollary.

Corollary 4.7. Consider the system defined as

$$
\begin{aligned}
\dot{x}(t) & =\mathcal{J} x(t), \\
u(t) & =\frac{1}{2}\left(f_{\partial}(t)+e_{\partial}(t)\right), \\
y(t) & =\frac{1}{2}\left(f_{\partial}(t)-e_{\partial}(t)\right),
\end{aligned}
$$

where $\left(f_{\partial}(t), e_{\partial}(t)\right)$ are the boundary port variable associated with $x(t)$; see Definition 3.5 .

The above system is a boundary control system with the associated semigroup a contraction. Furthermore, for $u \in C^{2}\left((0, \infty) ; \mathbb{R}^{n N}\right), x(0) \in H^{N}\left((a, b) ; \mathbb{R}^{n}\right)$, and $u(0)=\frac{1}{2}\left(f_{\partial}(0)+e_{\partial}(0)\right)$ we have that

$$
\frac{1}{2} \frac{d}{d t}\|x(t)\|^{2}=\|u(t)\|^{2}-\|y(t)\|^{2} .
$$

In the previous theorems we have seen that for the same Dirac structure the properties of the PDE, obtained by a choice of the inputs and outputs, can be completely different. Hence for the same underlying Dirac structure, many different system theoretic properties are possible. It is even possible that the PDE has no solution for the trivial input signal. Let us illustrate this situation in more detail in the following simple example.

Example 4.8. Consider the PDE on $[a, b]$

$$
\frac{\partial x}{\partial t}(t, z)=\frac{\partial x}{\partial z}(t, z) .
$$

Following section 3 , we see that $N=n=1$, and $P(1)=1$. The boundary port variables (see Definition 3.5) are

$$
\left(\begin{array}{l}
f_{\partial}(t) \\
e_{\partial}(t)
\end{array}\right)=\frac{1}{\sqrt{2}}\left(\begin{array}{l}
x(t, b)-x(t, a) \\
x(t, b)+x(t, a)
\end{array}\right) .
$$

A short calculation gives that the PDE as discussed in Theorem 4.2 is (4.40) with the boundary input

$$
u(t)=s \sqrt{2}[x(t, b)-v x(t, a)]
$$

and output

$$
y(t)=\frac{1}{\sqrt{2}}\left(\tilde{w}_{2}+\tilde{w}_{1}\right) x(t, b)+\frac{1}{\sqrt{2}}\left(\tilde{w}_{2}-\tilde{w}_{1}\right) x(t, a),
$$


where $s$ is a nonzero scalar, $v$ is an element of $[-1,1]$, and $\tilde{w}_{1}, \tilde{w}_{2}$ are such that $\tilde{w}_{2}(1+v)-\tilde{w}_{1}(1-v) \neq 0$. As shown in Theorem 4.2 for any choice of $v \in[-1,1]$, we have that the PDE (4.40) with input (4.41) and output (4.42) has a unique classical solution provided the initial condition and the input are sufficiently smooth. Although the underlying Dirac structure stays the same, the system theoretic properties may be different for different choices of $v$. For instance, if $v=1$, then the associated semigroup is unitary, whereas for $v=0$, the associated semigroup is zero for $t \geq(b-a)$; see also [31].

Now one may wonder which (linear combination) of the boundary port variables may serve as an input, by which we only mean that it may be chosen in some sufficiently large (linear) space. Note that the choice, $u(t)=f_{\partial}(t)-e_{\partial}(t)$, gives that the input is located at $z=a$. Since (4.40) represents the left shift, it may be clear that the value of $x$ at $a$ cannot be an input. Even more, for $u \equiv 0$, the PDE does not have a solution.

5. Port Hamiltonian system. In this section, we define port Hamiltonian systems associated with (constant) skew-symmetric matrix operators. These systems are defined in terms of network-based modeling [1, 18, 28] which is based on the definition of two objects: the interconnection structure defined by a Dirac structure and the Hamiltonian function representing the total energy of the system. First, using the definition of the Dirac structure associated with a skew-symmetric operator given in section 3, we define a port Hamiltonian system with boundary port variables. Second, using the results of section 4, we formulate these port Hamiltonian systems as boundary control systems. In subsection 5.2 we treat extensively the example of the Timoshenko beam.

5.1. Linear port Hamiltonian systems with boundary port variables. We now extend the definition of linear port Hamiltonian systems as defined for finitedimensional state spaces [28] to infinite-dimensional state spaces. The interconnection structure is defined by a Dirac structure associated with the skew-symmetric differential operator according to Theorem 3.6. The Hamiltonian function, generating this port Hamiltonian system, is defined by a coercive operator relating the state variable to the effort variable.

In the introductory example of the section 2, the skew-symmetric operator was the $2 \times 2$ matrix differential operator of differential order 1 corresponding to the canonical interdomain coupling, and the Dirac structure was the Stokes-Dirac structure. The symmetric operator was defined by the elasticity modulus and the mass distribution defining the elastodynamic energy of the string.

Definition 5.1. Consider the domain $Z=(a, b) \subset \mathbb{R}$. Let the space of flow variables $\mathcal{F}_{Z}$ be equal to $L^{2}\left((a, b) ; \mathbb{R}^{n}\right)$ and let the space of effort variables $\mathcal{E}_{Z}$ be equal to $\mathcal{F}_{Z}$. Consider an $n \times n$ matrix skew-symmetric differential operator of differential order $N$ denoted as $\mathcal{J}$ defined by (3.1) and (3.2). Define the bond space $\mathcal{B}=\mathcal{F}_{Z} \times \mathbb{R}^{n N} \times \mathcal{E}_{Z} \times \mathbb{R}^{n N}$ and the Dirac structure $\mathcal{D}_{\mathcal{J}}$ associated with the skewsymmetric differential operator $\mathcal{J}$ as defined in Theorem 3.6. Let $\mathcal{L}$ be a coercivesymmetric operator on $\mathcal{E}_{Z}$. The port Hamiltonian system with the boundary port variables associated with $\mathcal{J}$ and generated by $\mathcal{L}$ is defined by

$$
\left(\dot{x}(t), f_{\partial}(t), \mathcal{L} x(t), e_{\partial}(t)\right) \in \mathcal{D}_{\mathcal{J}}, \quad t \geq 0,
$$

where $\left(\begin{array}{c}f_{\partial} \\ e_{\partial}\end{array}\right)$ is the boundary port associated with $e:=\mathcal{L} x$ according to Definition 3.5.

REMARK 5.2. It may be noted that the system in Definition 5.1 corresponds to 
the abstract system $\dot{x}(t)=A x(t)$ defined by the differential operator

$$
A=\mathcal{J} \mathcal{L}
$$

which need not be skew-symmetric nor have constant coefficients.

It is also worth making explicit the Hamiltonian function representing the energy of the system

$$
H(x)=\frac{1}{2}\langle x, \mathcal{L} x\rangle,
$$

where $\langle\cdot, \cdot\rangle$ denotes the natural inner product on the space $\mathcal{E}_{Z}$. The port Hamiltonian system of section 4 may hence be seen as a particular case with $\mathcal{L}=I$.

Noting that $\frac{d H(x(t))}{d t}=\langle\dot{x}(t), \mathcal{L} x(t)\rangle$, by the definition of Dirac structure, one obtains the following energy balance equation:

$$
\frac{d H(x(t))}{d t}=\frac{1}{2}\left(f_{\partial}^{T}(t), e_{\partial}^{T}(t)\right) \Sigma\left(\begin{array}{l}
f_{\partial}(t) \\
e_{\partial}(t)
\end{array}\right) .
$$

This expresses that the variation of the energy of the boundary port Hamiltonian system is equal to the flow of energy at the boundary of the system's domain.

This also motivates us to take the state space equal to those $x$ for which the Hamiltonian is finite. Since $\mathcal{L}$ is coercive on $\mathcal{E}_{Z}=L^{2}\left((a, b) ; \mathbb{R}^{n}\right)$, we see that the state space $\mathcal{X}$ is $L^{2}\left((a, b) ; \mathbb{R}^{n}\right)$ with the new inner product

$$
\left\langle x_{1}, x_{2}\right\rangle_{\mathcal{X}}=\left\langle x_{1}, \mathcal{L} x_{2}\right\rangle_{L^{2}\left((a, b) ; \mathbb{R}^{n}\right)} .
$$

In the previous definition we have defined linear port Hamiltonian systems with boundary port variables using the definition of Dirac structure for which the port variables are not split into input and output variables. However, we have seen in section 4 that using a specific subspace of the port variables, one may define input and output variables as belonging to complementary subspaces of the boundary port variables. Moreover, by choosing in an appropriate way these subspaces, one may define a boundary control system with its associated semigroup being a contraction. In the following, we reformulate the boundary port Hamiltonian system of Definition 5.1 as a boundary control system. We use the parameterization of the input and output variables and the contractive semigroups associated with the Dirac structure $\mathcal{D}_{\mathcal{J}}$ given in section 4 . The state variables have become the image of the effort variables through the coercive operator $\mathcal{L}^{-1}$.

Theorem 5.3. The port Hamiltonian system of Definition 5.1 may be formulated as a boundary control system on the state space $\mathcal{X}$ :

$$
\left(\dot{x}(t), f_{\partial}(t), \mathcal{L} x(t), e_{\partial}(t)\right) \in \mathcal{D}_{\mathcal{J}}, \quad t \geq 0,
$$

with the input variables defined by choosing some full rank matrix $W$ of size $n N \times 2 n N$ satisfying (4.1) and the map

$$
\mathcal{B} x(t)=W\left(\begin{array}{l}
f_{\partial}(t) \\
e_{\partial}(t)
\end{array}\right)=u(t)
$$

on the domain

$$
D(\mathcal{B})=D(\mathcal{J}) .
$$


Furthermore, define the port conjugated output

$$
y(t)=\tilde{W}\left(\begin{array}{l}
f_{\partial}(t) \\
e_{\partial}(t)
\end{array}\right)
$$

with $\tilde{W}$ a full rank matrix of size $n N \times 2 n N$ with $(\underset{\tilde{W}}{W})$ invertible. Then for $u \in$ $C^{2}\left((0, \infty) ; \mathbb{R}^{n N}\right), x(0) \in H^{N}\left((a, b), \mathbb{R}^{n}\right)$, and $u(0)=\mathcal{B} x(0)$, the following balance equation is satisfied:

$$
\frac{d}{d t} H(x(t))=\frac{1}{2}\left(u^{T}(t) \quad y^{T}(t)\right) P_{W, \tilde{W}}\left(\begin{array}{l}
u(t) \\
y(t)
\end{array}\right),
$$

where $P_{W, \tilde{W}}$ is defined in (4.13).

The proof is a straightforward extension of the proof of Theorem 4.1 using the following lemma.

Lemma 5.4. Assume that $W$ satisfies (4.1). The differential operator $A_{W}=\mathcal{J} \mathcal{L}$ with the domain $D\left(A_{W}\right)=\left\{x \in \mathcal{X} \mid \mathcal{L} x \in D\left(J_{W}\right)\right\}$ (see (4.4)) generates a contraction semigroup on $\mathcal{X}$.

Proof. We first show that $A_{W}$ is dissipative. For $x \in D\left(A_{W}\right)$, we have that

$$
\left\langle x, A_{W} x\right\rangle_{\mathcal{X}}=\langle x, \mathcal{J} \mathcal{L} x\rangle_{\mathcal{X}}=\langle x, \mathcal{L} \mathcal{J} \mathcal{L} x\rangle_{L_{2}}=\langle e, \mathcal{J} e\rangle_{L_{2}},
$$

where $e=\mathcal{L} x$. Since $e \in D\left(J_{W}\right)$ and since $J_{W}$ is a restriction of $\mathcal{J}$, we find that

$$
\left\langle x, A_{W} x\right\rangle_{\mathcal{X}}=\left\langle e, J_{W} e\right\rangle_{L_{2}},
$$

which is nonpositive, since $J_{W}$ generates a contraction semigroup on $L^{2}\left((a, b) ; \mathbb{R}^{n}\right)$.

It is not hard to show that $A_{W}^{*}=J_{W}^{*} \mathcal{L}$ with $D\left(A_{W}^{*}\right)=\left\{x \in \mathcal{X} \mid \mathcal{L} x \in D\left(J_{W}^{*}\right)\right\}$. Using an argument similar to that above, we find that on $D\left(A_{W}^{*}\right)$

$$
\left\langle x, A_{W}^{*} x\right\rangle_{\mathcal{X}} \leq 0 .
$$

Hence we conclude that $A_{W}$ generates a contraction semigroup on $\mathcal{X}$.

5.2. Example: The Timoshenko's beam model. Timoshenko's beam model describes the infinitesimal planar deformations of a flexible beam reduced to its neutral fiber with some particular geometrical assumptions. We briefly recall the Hamiltonian formulation as proposed by Golo, Talasila, and van der Schaft [6]. Note that this corresponds to taking the Legendre transform of the usual Lagrangian formulation. Consider the spatial domain $Z=[a, b]$. Denote the angular displacement by $q_{\theta}$, the transversal displacement of the beam by $q_{y}$, and the conjugated momenta by $p_{\theta}$ and $p_{y}$. The elastic potential energy density is given by $\mathcal{U}(q)=\frac{1}{2} \int_{Z} F^{T} q d z$, where the strain wrench (torque and force) is $F=K q$. Let $K=\operatorname{diag}\left(c_{\theta}, c_{y}\right)$ denote the positive definite compliance matrix which depends on the elasticity properties of the material and its geometry. The kinetic energy is given by $\mathcal{K}(p)=\frac{1}{2} \int_{Z} v^{T} p d z$, where the coenergy variable is the velocity $v=M^{-1} p$. $M$ denotes the positive definite inertia matrix which is given as $M=\operatorname{diag}(\iota, \mu)$ with $\iota$ the momentum of inertia of the beam per unit length and $\mu$ the mass per unit length. It is immediate that $F=\delta_{q} \mathcal{U}(q)$ and $v=\delta_{p} \mathcal{K}(q)$, where $\delta$ denotes the variational derivative [20].

Choose the state vector $x$ as

$$
x=\left(\begin{array}{l}
q_{\theta} \\
q_{y} \\
p_{\theta} \\
p_{y}
\end{array}\right)=\left(\begin{array}{l}
q \\
p
\end{array}\right) .
$$


The Timoshenko beam model may be expressed as the following Hamiltonian evolution equations $[6,7]$ :

$$
\frac{\partial x}{\partial t}=\mathcal{J}\left(\begin{array}{c}
\frac{\partial \mathcal{H}}{\partial q} \\
\frac{\partial \mathcal{H}}{\partial p}
\end{array}\right),
$$

where $\mathcal{H}(q, p)=\mathcal{U}(q)+\mathcal{K}(p)$ is the total elastodynamic energy of the beam, and the skew-symmetric differential operator $\mathcal{J}$ is

$$
\mathcal{J}=\left(\begin{array}{cc}
0_{2} & \left(\begin{array}{cc}
\frac{\partial}{\partial z} & 0 \\
-1 & \frac{\partial}{\partial z}
\end{array}\right) \\
\left(\begin{array}{cc}
\frac{\partial}{\partial z} & 1 \\
0 & \frac{\partial}{\partial z}
\end{array}\right) & 0_{2}
\end{array}\right) .
$$

We now derive the port Hamiltonian formulation of this system. The time variation of the energy variables is defined as flow variables:

$$
\frac{\partial}{\partial t}\left(\begin{array}{l}
q \\
p
\end{array}\right):=\left(\begin{array}{l}
f_{q} \\
f_{p}
\end{array}\right)
$$

The variational derivative of the total energy $\delta_{x} \mathcal{H}$ defines the effort variables:

$$
\left(\begin{array}{l}
e_{q} \\
e_{p}
\end{array}\right):=\mathcal{L}\left(\begin{array}{c}
q \\
p
\end{array}\right)=\left(\begin{array}{cc}
K & 0 \\
0 & M^{-1}
\end{array}\right)\left(\begin{array}{l}
q \\
p
\end{array}\right) .
$$

Note that

$$
\mathcal{L}\left(\begin{array}{c}
q \\
p
\end{array}\right)=\left(\begin{array}{c}
\frac{\partial \mathcal{H}}{\partial q} \\
\frac{\partial \mathcal{H}}{\partial p}
\end{array}\right)
$$

More precisely,

$$
e_{q}=\left(\begin{array}{cc}
c_{\theta} & 0 \\
0 & c_{y}
\end{array}\right)\left(\begin{array}{c}
q_{\theta} \\
q_{y}
\end{array}\right)=\left(\begin{array}{c}
T \\
F_{y}
\end{array}\right)
$$

is the vector composed of the torque and the force, and

$$
e_{p}=\left(\begin{array}{cc}
\iota^{-1} & 0 \\
0 & \mu^{-1}
\end{array}\right)\left(\begin{array}{c}
p_{\theta} \\
p_{y}
\end{array}\right)=\left(\begin{array}{c}
\omega \\
v_{y}
\end{array}\right)
$$

is the vector composed of the angular and longitudinal velocities.

Hence, according to the evolution equation (5.9), the flow variables are related to the coenergy variables by the skew-symmetric differential operator $\mathcal{J}$ defined in (3.1)

$$
\left(\begin{array}{l}
f_{q} \\
f_{p}
\end{array}\right)=\mathcal{J}\left(\begin{array}{l}
e_{q} \\
e_{p}
\end{array}\right) .
$$

This differential operator may be written as

$$
\mathcal{J}=P(0)+P(1) \frac{\partial}{\partial z},
$$


where

$$
P(0)=\left(\begin{array}{cc}
0_{2} & \left(\begin{array}{cc}
0 & 0 \\
-1 & 0
\end{array}\right) \\
\left(\begin{array}{ll}
0 & 1 \\
0 & 0
\end{array}\right) & 0_{2}
\end{array}\right), \quad P(1)=\left(\begin{array}{cc}
0_{2} & \left(\begin{array}{ll}
1 & 0 \\
0 & 1
\end{array}\right) \\
\left(\begin{array}{ll}
1 & 0 \\
0 & 1
\end{array}\right) & 0_{2}
\end{array}\right)
$$

The symmetric matrix $Q$ corresponding to the bilinear term on the boundary variables in Theorem 3.1 and given in (3.5) reduces to $Q=P(1)$. The matrix $R_{\text {ext }}$ defining the boundary port variables equals (see (3.7))

$$
R_{\text {ext }}=\frac{\sqrt{2}}{2}\left(\begin{array}{cccccccc}
0 & 0 & 1 & 0 & 0 & 0 & -1 & 0 \\
0 & 0 & 0 & 1 & 0 & 0 & 0 & -1 \\
1 & 0 & 0 & 0 & -1 & 0 & 0 & 0 \\
0 & 1 & 0 & 0 & 0 & -1 & 0 & 0 \\
1 & 0 & 0 & 0 & 1 & 0 & 0 & 0 \\
0 & 1 & 0 & 0 & 0 & 1 & 0 & 0 \\
0 & 0 & 1 & 0 & 0 & 0 & 1 & 0 \\
0 & 0 & 0 & 1 & 0 & 0 & 0 & 1
\end{array}\right)
$$

According to Definition 3.5 the port variables are

$$
\left(\begin{array}{l}
f_{\partial} \\
e_{\partial}
\end{array}\right)=R_{\operatorname{ext}}\left(\begin{array}{cc}
\mathcal{L} & 0 \\
0 & \mathcal{L}
\end{array}\right)\left(\begin{array}{l}
q(b) \\
p(b) \\
q(a) \\
p(a)
\end{array}\right)
$$

Considering relations (5.11), (5.12), and (5.13),

$$
\left(\begin{array}{c}
f_{\partial} \\
e_{\partial}
\end{array}\right)=\frac{\sqrt{2}}{2}\left(\begin{array}{c}
\omega(b)-\omega(a) \\
v_{y}(b)-v_{y}(a) \\
T(b)-T(a) \\
F_{y}(b)-F_{y}(a) \\
T(b)+T(a) \\
F_{y}(b)+F_{y}(a) \\
\omega(b)+\omega(a) \\
v_{y}(b)+v_{y}(a)
\end{array}\right)
$$

The associated Dirac structure is given by

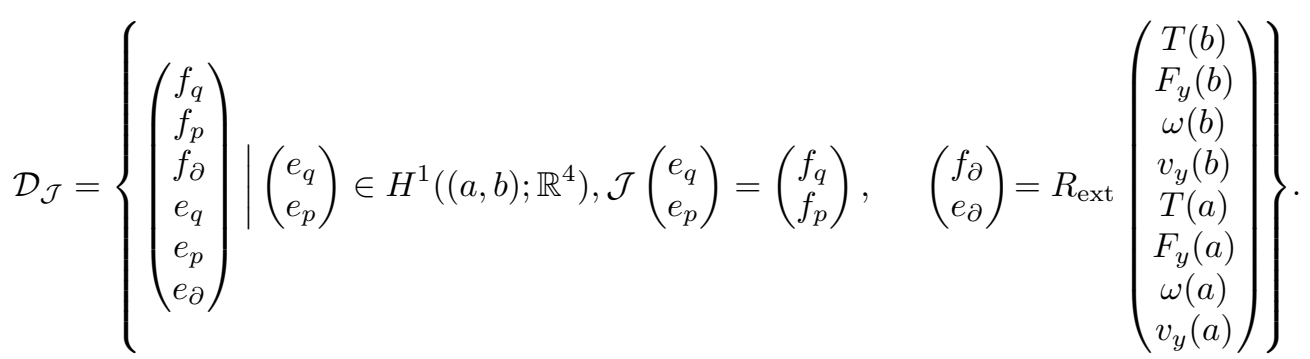

We now illustrate the derivation of boundary control systems from the port Hamiltonian system using two different choices of the matrix $W$ defining them according to Theorem 5.3. The first choice corresponding to the boundary control system is 
associated with a unitary semigroup and in the other choice corresponds to a system in the scattering representation.

For the unitary case let us choose the matrix $W$ given in (4.2) with the invertible matrix $S$ and matrix $V$ satisfying $V V^{T}=I$ chosen as follows:

$$
S=\frac{1}{2 \sqrt{2}}\left(\begin{array}{cc}
-I_{2} & I_{2} \\
I_{2} & I_{2}
\end{array}\right) \text { and } V=\left(\begin{array}{cc}
0 & I_{2} \\
-I_{2} & 0
\end{array}\right)
$$

This choice corresponds to define the inputs

$$
\begin{aligned}
u & =S\left(I_{4}+V \quad I_{4}-V\right)\left(\begin{array}{c}
f_{\partial} \\
e_{\partial}
\end{array}\right) \\
& =\frac{1}{\sqrt{2}}\left(\begin{array}{cccc}
-I_{2} & 0 & 0 & I_{2} \\
0 & I_{2} & I_{2} & 0
\end{array}\right)\left(\begin{array}{c}
f_{\partial} \\
e_{\partial}
\end{array}\right)=\left(\begin{array}{c}
\omega(a) \\
v_{y}(a) \\
T(b) \\
F_{y}(b)
\end{array}\right) .
\end{aligned}
$$

The unitary semigroup associated with the boundary control $u=0$ corresponds to the following boundary conditions:

$$
\omega(a, t)=v_{y}(a, t)=M(b, t)=F_{y}(b, t)=0,
$$

which are the so-called clamped-free boundary conditions. According to Theorem 4.4 the output conjugated to this input is

$$
y=\tilde{S}\left(I_{4}+\tilde{V} \quad I_{4}-\tilde{V}\right)\left(\begin{array}{l}
f_{\partial} \\
e_{\partial}
\end{array}\right)
$$

with $\tilde{V}$ unitary, $\tilde{S}$ invertible, and

$$
2 \tilde{S}\left(I_{4}-\tilde{V} V^{T}\right) S=I_{4} .
$$

For example, choosing $\tilde{V}=-V=\left(\begin{array}{cc}0 & -I_{2} \\ I_{2} & 0\end{array}\right)$ and $\tilde{S}=S=\frac{1}{2 \sqrt{2}}\left(\begin{array}{cc}-I_{2} & I_{2} \\ I_{2} & I_{2}\end{array}\right)$ we obtain

$$
y=\left(\begin{array}{c}
-T(a) \\
-F_{y}(a) \\
\omega(b) \\
v_{y}(b)
\end{array}\right)
$$

For the contractive case, let us choose the matrix $W$ given in (4.2) with the invertible matrix $S$ and matrix $V$, satisfying $V V^{T} \leq I$, chosen as follows:

$$
S=\frac{\sqrt{2}}{4}\left(\begin{array}{cc}
I_{2} & I_{2} \\
-I_{2} & I_{2}
\end{array}\right) \text { and } V=\left(\begin{array}{cc}
0 & 0 \\
0 & 0
\end{array}\right)
$$

According to Theorem 5.3 the inputs are

$$
u=\frac{\sqrt{2}}{4}\left(\begin{array}{cccc}
I_{2} & I_{2} & I_{2} & I_{2} \\
-I_{2} & I_{2} & -I_{2} & I_{2}
\end{array}\right)\left(\begin{array}{c}
f_{\partial} \\
e_{\partial}
\end{array}\right)=\frac{1}{2}\left(\begin{array}{c}
\omega(b)+T(b) \\
v_{y}(b)+F_{y}(b) \\
\omega(a)-T(a) \\
v_{y}(a)-F_{y}(a)
\end{array}\right) .
$$


For $\tilde{S}$ (see Theorem 4.6) we choose $S$ and so the outputs are

$$
y=\frac{\sqrt{2}}{4}\left(\begin{array}{cccc}
I_{2} & I_{2} & -I_{2} & -I_{2} \\
-I_{2} & I_{2} & I_{2} & -I_{2}
\end{array}\right)\left(\begin{array}{l}
f_{\partial} \\
e_{\partial}
\end{array}\right)=-\frac{1}{2}\left(\begin{array}{c}
\omega(a)+T(a) \\
v_{y}(a)+F_{y}(a) \\
\omega(b)-T(b) \\
v_{y}(b)-F_{y}(b)
\end{array}\right) .
$$

In this case, the boundary inputs and outputs correspond to the scattering variables and $\frac{1}{2}\|x(t)\|_{\mathcal{X}}^{2}=\|u(t)\|^{2}-\|y(t)\|^{2}$.

6. Conclusion and further work. The work presented in this paper relates the structure of a class of linear infinite-dimensional dynamical models induced by the physical modeling (existence of energy function, power continuous interconnection structure) with system theoretical properties (passivity, etc.). More precisely, we have defined a class of infinite-dimensional linear systems associated with skew-symmetric differential operators and we have related them to boundary control systems. Knowing the underlying physical structure and the system theoretical notions will be very useful in the further analysis and design for our class of infinite-dimensional systems, for instance, in the construction of stabilizing feedbacks.

Therefore, we have, in the first instance, defined a Dirac structure on a Hilbert space associated with skew-symmetric differential operators with constant coefficients. Using the Stokes theorem, we have defined port boundary variables as the image of the boundary values under a linear map, which is derived from the differential operator. Then we have shown that the differential operator together with the boundary port variables defines a Dirac structure on a vector space (the space of bond variables) endowed with a canonical symmetric pairing. This defines the geometrical structure associated with the initial PDE.

In the second instance, we have shown that one may derive from the Dirac structure infinitesimal generators of contraction semigroups. These infinitesimal generators are obtained by restricting the domain of the skew-symmetric operator to parameterized subspaces. More precisely, we have shown that we have obtained a parameterization of all the contraction semigroups which are associated with the skew-symmetric operator.

In the third instance, we have derived a formulation of our class of infinitedimensional systems as boundary control systems associated with the class of contraction semigroups obtained from the Dirac structure. We have defined outputs conjugated to the inputs of the boundary control systems in such a way that the system satisfies a power balance equation in a way similar to dissipative systems [25].

In the fourth instance, these results are used to define infinite-dimensional port Hamiltonian systems. These systems are defined with respect to the Dirac structure associated with a skew-symmetric differential operator and a coercive operator defining the Hamiltonian functional, i.e., the total energy of the system. Again from such a port Hamiltonian system one may derive a class of boundary control system associated with contraction semigroups. This is illustrated by the example of Timoshenko's beam.

A natural question is the relation of our class of systems, especially the Hamiltonian systems with the systems nodes (see [26]), and with the class of well-posed linear systems. This has been partially done in [31] for the system nodes and in [32] for well-posed systems (using the idea of feedback). Another issue is the generalization of this work to PDEs on an $n$-dimensional spatial domain. 
Finally, this works also opens the way for the generalization to infinite-dimensional systems of the synthesis of stabilizing controllers using the immersion and Hamiltonian reduction proposed in [13, 22].

\section{Appendix. Technical lemmas.}

Lemma A.1. Let $W$ be an $n N \times 2 n N$ matrix and let $\Sigma=\left(\begin{array}{ll}0 & I \\ I & 0\end{array}\right)$. Then $W$ has rank $n N$ and $W \Sigma W^{T} \geq 0$ if and only if there exist a matrix $V \in \mathbb{R}^{n N \times n N}$ and an invertible matrix $S \in \mathbb{R}^{n N \times n N}$ such that

$$
W=S(I+V \quad I-V)
$$

with $V V^{T} \leq I$.

Furthermore, $W \Sigma W^{T}=0$ if and only if $V$ is unitary.

Proof. If $W$ is of the form (A.1), then we find

$$
W \Sigma W^{T}=S(I+V \quad I-V) \Sigma\left(\begin{array}{c}
I+V^{T} \\
I-V^{T}
\end{array}\right) S^{T}=S\left[2 I-2 V V^{T}\right] S^{T},
$$

which is nonnegative, since $V V^{T} \leq I$.

Now we prove that if $W$ is of full rank and is such that $W \Sigma W^{T} \geq 0$, then (A.1) holds. Writing $W$ as $W=\left(\begin{array}{ll}W_{1} & W_{2}\end{array}\right)$, we have that $W \Sigma W^{T} \geq 0$ is equivalent to $W_{1} W_{2}^{T}+W_{2} W_{1}^{T} \geq 0$. Hence

$$
\left(W_{1}+W_{2}\right)\left(W_{1}+W_{2}\right)^{T} \geq\left(W_{1}-W_{2}\right)\left(W_{1}-W_{2}\right)^{T} \geq 0 .
$$

If $x \in \operatorname{ker}\left(\left(W_{1}+W_{2}\right)^{T}\right)$, then the above inequality implies that $x \in \operatorname{ker}\left(\left(W_{1}-W_{2}\right)^{T}\right)$. Thus $x \in \operatorname{ker}\left(W_{1}^{T}\right) \cap \operatorname{ker}\left(W_{2}^{T}\right)$. Since $W$ has full rank, this implies that $x=0$. Hence $W_{1}+W_{2}$ is invertible.

Using (A.2) once again, we see that

$$
\left(W_{1}+W_{2}\right)^{-1}\left(W_{1}-W_{2}\right)\left(W_{1}-W_{2}\right)^{T}\left(W_{1}+W_{2}\right)^{-T} \leq I
$$

and thus $V:=\left(W_{1}+W_{2}\right)^{-1}\left(W_{1}-W_{2}\right)$ satisfies $V V^{T} \leq I$. Summarizing, we have

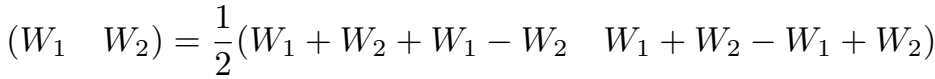

$$
\begin{aligned}
& =\frac{1}{2}\left(W_{1}+W_{2}\right)(I+V \quad I-V) .
\end{aligned}
$$

Defining $S:=\frac{1}{2}\left(W_{1}+W_{2}\right)$, we have shown the representation (A.1).

If instead of inequality we have equality for $W$, then it is easy to show that we have equality in the equation for $V$ as well. Thus $V$ is unitary.

LEMMA A.2. Suppose that the $n N \times 2 n N$ matrix $W$ can be written in the format of (A.1), i.e., $W=S(I+V \quad I-V)$ with $S$ and $V$ square matrices, and $S$ is invertible. Then the kernel of $W$ equals the range of $\left(\begin{array}{c}I-V \\ -I-V\end{array}\right)$.

If $V$ is unitary, then the kernel of $W$ equals the range of $\Sigma W^{T}$.

Proof. Let $\left(\begin{array}{l}x_{1} \\ x_{2}\end{array}\right)$ be in the range of $\left(\begin{array}{c}I-V \\ -I-V\end{array}\right)$. By equality (A.1), we have that

$$
\begin{aligned}
W\left(\begin{array}{l}
x_{1} \\
x_{2}
\end{array}\right) & =S\left(\begin{array}{ll}
I+V & I-V
\end{array}\right)\left(\begin{array}{c}
x_{1} \\
x_{2}
\end{array}\right) \\
& =S\left(\begin{array}{ll}
I+V & I-V
\end{array}\right)\left(\begin{array}{c}
I-V \\
-I-V
\end{array}\right) l=0 .
\end{aligned}
$$


Hence we see that the range of $\left(\begin{array}{c}I-V \\ -I-V\end{array}\right)$ lies in the kernel of $W$. It is easy to show that $W$ has rank $n N$, and so the kernel of $W$ has dimension $n N$. Thus, if we can show that the $2 n N \times n N$ matrix $\left(\begin{array}{c}I-V \\ -I-V\end{array}\right)$ has full rank, then we have proved the first assertion. If this matrix would not have full rank, then there should be a nontrivial element in its kernel. It is easy to see that the kernel consists of zero only, and so we have proved the first part of the lemma.

Suppose now that $V$ is unitary, then

$$
\left(\begin{array}{c}
I-V \\
-I-V
\end{array}\right)=\left(\begin{array}{c}
-I+V^{T} \\
-I-V^{T}
\end{array}\right) V=-\Sigma W^{T} S^{-T} V
$$

Since the range of $\Sigma W^{T}$ equals the range of $-\Sigma W^{T} S^{-T} V$, we have proved the second assertion.

Lemma A.3. Given the interval $[a, b]$ and a positive number $N \in \mathbb{N}$. There exist polynomials $f_{l j}(z), f_{r j}(z), j=0, \ldots, N-1$, such that

$$
\begin{gathered}
\frac{d^{k} f_{l, j}}{d z^{k}}(a)=\delta_{k j}, \quad k=0, \ldots, N-1, \\
\frac{d^{k} f_{l, j}}{d z^{k}}(b)=0, \quad k=0, \ldots, N-1,
\end{gathered}
$$

and

$$
\begin{gathered}
\frac{d^{k} f_{r, j}}{d z^{k}}(a)=0, \quad k=0, \ldots, N-1, \\
\frac{d^{k} f_{r, j}}{d z^{k}}(b)=\delta_{k j}, \quad k=0, \ldots, N-1 .
\end{gathered}
$$

Proof. Since the construction of $f_{r, j}$ is very similar to that of $f_{l, j}$, we show only how $f_{l, j}$ is constructed. These functions are constructed using backward induction. It is easily seen that

$$
f_{l, N-1}(z):=\frac{1}{(N-1) !}(z-a)^{N-1}(z-b)^{N} \frac{1}{(a-b)^{N}}
$$

satisfies condition (A.3). Suppose next that we have constructed the functions $f_{l, j}(z)$ for $j=j_{0}+1, \ldots, N-1$. We next construct $f_{l, j_{0}}(z)$. Define $\tilde{f}_{l, j_{0}}(z)$ as

$$
\tilde{f}_{l, j_{0}}(z)=\frac{1}{j_{0} !}(z-a)^{j_{0}}(z-b)^{N} \frac{1}{(a-b)^{N}} .
$$

It is easy to see that

$$
\frac{d^{k} \tilde{f}_{l, j_{0}}}{d z^{k}}(a)=\delta_{k j_{0}}, \quad k=0, \ldots, j_{0},
$$

and

$$
\frac{d^{k} \tilde{f}_{l, j_{0}}}{d z^{k}}(b)=0, \quad k=0, \ldots, N-1 .
$$

If we define the function $f_{l, j_{0}}(z)$ as

$$
f_{l, j_{0}}(z)=\tilde{f}_{l, j_{0}}(z)-\sum_{i=j_{0}+1}^{N-1} \frac{d^{i} f_{l, j_{0}}}{d z^{i}}(a) f_{l, i}(z),
$$

then it is straightforward to see that it satisfies (A.3). 


\section{REFERENCES}

[1] P.C. Breedveld, Physical Systems Theory in Terms of Bond Graphs, Ph.D. thesis, Technische Hogeschool Twente, Enschede, The Netherlands, 1984.

[2] T.J. Courant, Dirac manifolds, Trans. Amer. Math. Soc., 319 (1990), pp. 631-661.

[3] R.F. Curtain and H.J. Zwart, An Introduction to Infinite-Dimensional Linear System Theory, 1st ed., Springer-Verlag, Berlin, 1995.

[4] M. Dalsmo And A.J. VAn DeR Schaft, On representations and integrability of mathematical structures in energy-conserving physical systems, SIAM J. Control Optim., 37 (1999), pp. 54-91.

[5] I. Dorfman, Dirac Structures and Integrability of Nonlinear Evolution Equations, Wiley, New York, 1993.

[6] G. Golo, V. Talasila, and A.J. van der Schaft, A Hamiltonian formulation of the Timoshenko beam, in Proceedings of the Mechatronics, Drebbel Institute for Mechatronics, University of Twente, Enschede, The Netherlands, 2002, pp. 544-553. Full paper on accompanying CD-ROM.

[7] G. Golo, Interconnection Structures in Port-Based Modelling: Tools for Analysis and Simulation, Ph.D. thesis, University of Twente, Enschede, The Netherlands, 2002.

[8] G. Golo, O.V. Iftime, H. Zwart, and A.J. van der Schaft, Tools for Analysis of Dirac Structures on Hilbert Spaces, Memorandum Faculteit TW 1729, Universiteit Twente, Enschede, 2004, http://www.math.utwente.nl/ssb/annrep04/publs04.htm.

[9] V.I. Gorbachuk and M.L. Gorbachuk, Boundary Value Problems for Operator Differential Equations, Math. Appl. (Soviet Ser.) 48, Kluwer Academic Publishers, Norwell, MA, 1991. (Expanded and revised translation of Granichnye zadachi dia differentsial'no-operatornykh uravneii.)

[10] Y. Le Gorrec, H. Zwart, and B.M. Maschke, Dirac Structures and Boundary Control Systems Associated with Skew-Symmetric Differential Operators, Memorandum Faculteit TW 1730, Universiteit Twente, Enschede, 2004, http://www.math.utwente.nl/ssb/ annrep04/publs04.htm.

[11] D.C. Karnopp, D.L. Margolis, and R.C. Rosenberg, System Dynamics: A Unified Approach, Wiley, New York, 1990.

[12] B.M. Maschke, Interconnection and structure in physical systems' dynamics, in Proceedings of the 5th NOLCOS, NOLCOS'98, Enschede, 1998, pp. 291-296.

[13] B.M. Maschke, R. ORTEga, ANd A.J. van Der Schaft, Energy-based Lyapunov functions for forced Hamiltonian systems with dissipation, IEEE Trans. Automat. Control, 45 (2000), pp. 1498-1502.

[14] B.M. Maschke And A.J. van Der Schaft, Port controlled Hamiltonian systems: Modeling origins and system theoretic properties, in Proceedings of the 3rd NOLCOS, NOLCOS'92, Bordeaux, 1992, pp. 282-288.

[15] B.M. Maschke And A.J. VAn Der Schaft, Interconnected mechanical systems, Part 2: The dynamics of spatial mechanical networks, in Modelling and Control of Mechanical Systems, Imperial College Press, London, 1997, pp. 17-30.

[16] B.M. Maschke And A.J. VAn Der Schaft, Port controlled Hamiltonian representation of distributed parameter systems, in Workshop on Modeling and Control of Lagrangian and Hamiltonian Systems, Princeton, NJ, 2000.

[17] B.M. MASChKE AND A.J. VAN DER SChAFT, Compositional modelling of distributed-parameter systems, in Advanced Topics in Control Systems Theory, Lecture Notes in Control and Inform. Sci. 311, F. Lamnabhi-Lagarrigue, A. Loria, and E. Panteley, eds., Springer-Verlag, Berlin, 2005, pp. 115-154.

[18] B.M. Maschke, A.J. van der Schaft, and P.C. Breedveld, An intrinsic Hamiltonian formulation of network dynamics: Non-standard poisson structures and gyrators, J. Franklin Inst., 329 (1992), pp. 923-966.

[19] B.M. MASChKE AND A.J. VAN DER SCHAFT, Canonical interdomain coupling in distributed parameter systems: An extension of the symplectic gyrator, in Proceedings of the International Mechanical Engineering Congress and Exposition, New York, 2001.

[20] P.J. Olver, Applications of Lie groups to differential equations, 2nd ed., Grad. Texts in Math. 107, Springer-Verlag, New York, 1993.

[21] R. Ortega, A.J. van der Schaft, I. Mareels, and B. Maschke, Putting energy back in control, IEEE Control Syst. Mag., 21 (2001), pp. 18-32.

[22] R. Ortega, A.J. van Der Schaft, B. Maschke, And G. Escobar, Interconnection and damping assignment: Passivity-based control of port-controlled Hamiltonian systems, Automatica, 38 (2002), pp. 585-596. 
[23] A. Parsian and A. Shafei Deh Abad, Dirac structures on Hilbert spaces, Int. J. Math. Math. Sci., 22 (1999), pp. 97-108.

[24] R.S. Phillips, Dissipative operators and hyperbolic systems of partial differential equations, Trans. Amer. Math. Soc., 90 (1959), pp. 193-254.

[25] H.K. Pillai and J. Willems, Lossless and dissipative distributed systems, SIAM J. Control Optim., 40 (2002), pp. 1406-1430.

[26] O.J. Staffans, Well-Posed Linear Systems, Cambridge University Press, London, 2005.

[27] A.J. VAN DER Schaft, $L_{2}$-Gain and Passivity Techniques in Nonlinear Control, 2nd revised and enlarged ed., Comm. Control Engrg. Ser., Springer-Verlag, London, 1999; 1st ed., Lecture Notes in Control and Inform. Sci. 218, Springer-Verlag, Berlin, 1996.

[28] A.J. VAn DER Schaft AND B.M. MaschKe, The Hamiltonian formulation of energy conserving physical systems with external ports, Archiv. Elektronik Übertragungstechnik, 49 (1995), pp. 362-371.

[29] A.J. VAn Der Schaft And B.M. Maschke, Interconnected mechanical systems, Part 1: Geometry of interconnection and implicit Hamiltonian systems, in Modelling and Control of Mechanical Systems, Imperial College Press, London, 1997, pp. 1-16.

[30] A.J. VAn DeR SChafT AND B.M. MaschKe, Hamiltonian formulation of distributed parameter systems with boundary energy flow, J. Geom. Phys., 42 (2002), pp. 166-174.

[31] J.A. Villegas, Y. Le Gorrec, H. Zwart, and A.J. van der Schaft, Boundary control systems and the system nodes, in 16th IFAC World Congress, Praha, 2005.

[32] H. Zwart, Y. Le Gorrec, B.M. Maschke, and J.A. Villegas, Well-posedness of a class of boundary control systems, in 44th IEEE CDC-ECC, Seville, Spain, submitted. 